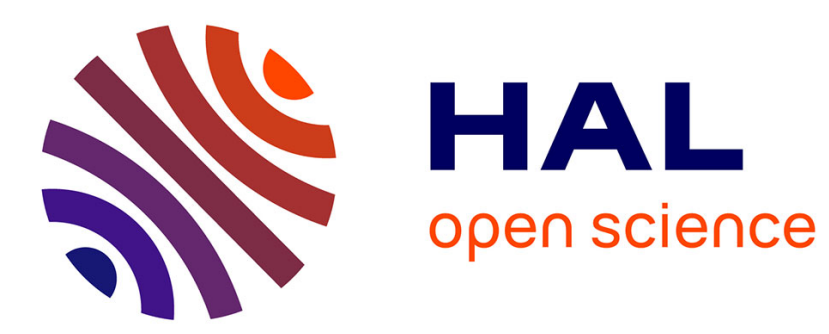

\title{
A domain derivative-based method for solving elastodynamic inverse obstacle scattering problems
}

Frédérique Le Louër

\section{To cite this version:}

Frédérique Le Louër. A domain derivative-based method for solving elastodynamic inverse obstacle scattering problems. Inverse Problems, 2015, 31 (11). hal-01152319v2

\section{HAL Id: hal-01152319 \\ https://hal.science/hal-01152319v2}

Submitted on 17 Aug 2015

HAL is a multi-disciplinary open access archive for the deposit and dissemination of scientific research documents, whether they are published or not. The documents may come from teaching and research institutions in France or abroad, or from public or private research centers.
L'archive ouverte pluridisciplinaire HAL, est destinée au dépôt et à la diffusion de documents scientifiques de niveau recherche, publiés ou non, émanant des établissements d'enseignement et de recherche français ou étrangers, des laboratoires publics ou privés. 


\title{
A domain derivative-based method for solving elastodynamic inverse obstacle scattering problems
}

\author{
Frédérique Le Louër *
}

\begin{abstract}
The present work is concerned with the shape reconstruction problem of isotropic elastic inclusions from far-field data obtained by the scattering of a finite number of time-harmonic incident plane waves. This paper aims at completing the theoretical framework which is necessary for the application of geometric optimization tools to the inverse transmission problem in elastodynamics. The forward problem is reduced to systems of boundary integral equations following the direct and indirect methods initially developed for solving acoustic transmission problems. We establish the Fréchet differentiability of the boundary to farfield operator and give a characterization of the first Fréchet derivative and its adjoint operator. Using these results we propose an inverse scattering algorithm based on the iteratively regularized Gauß-Newton method and show numerical experiments in the special case of star-shaped obstacles.
\end{abstract}

Keywords : Elastic scattering, penetrable obstacle, boundary integral equation system, Fréchet derivative, regularized Newton type method.

\section{Introduction}

This paper is concerned with the shape reconstruction problem of a three-dimensional bounded penetrable obstacle from far-field data obtained by the scattering of time-harmonic incident waves in elastodynamics. Efficient solution method for this inverse problem is of practical interest for various physical applications such as non destructive testing or geophysical exploration.

In the last two decades, numerous new techniques have been developed for solving shape reconstruction problems. Most attention has been spent on the following two categories of approaches: qualitative methods and nonlinear optimization methods. Examples of qualitative methods are the Linear Sampling Method [12, 13] and the Factorization Method [39] which have been developed initially for solving inverse acoustic scattering problems in exterior domains. One can find in the scientific literature numerous extensions of these qualitative methods to many different configurations : a non-exhaustive list of papers in linear elasticity includes inverse scattering in unbounded domains $[10,11]$, in half-spaces [4], in waveguides $[6,7]$ and in periodic structures [34]. These inverse scattering algorithms are very efficient for physical applications which do not seek to recover additional informations on the scattering object (i.e. material properties). The main disadvantage of sampling methods is that it requires, especially in unbounded media, a knowledge of the full far-field patterns for all directions of incidence and observation. Uniqueness results are also based on such criteria [22] excepted for balls or convex polyhedral scatterers that can be determined from far-field data obtained by the scattering of a single incident plane wave [33]. The inverse problem of recovering the shape of an obstacle from measurements obtained by the scattering a finite number of incident waves can be numerically solved by nonlinear optimization algorithms based either on geometric optimization tools or on topological optimization tools. In this case the inverse problem is often formulated as a nonlinear least squares problem for which iterative algorithm can be

\footnotetext{
*Sorbonne Université, Université de technologie de Compiègne, LMAC EA2222 Laboratoire de Mathématiques Appliquées de Compiègne - CS 60319 - 60203 Compiègne cedex, France, frederique.le-louer@utc.fr
} 
applied to recover an approximate solution. Geometric optimization consists in minimizing the nonlinear least square among a family of parametrized boundaries with same genus. The problem being severely ill-posed, the least squares are regularized by a quadratic penalty term, the so-called Tyckonov regularization. Pioneer work in this area were conducted by Kirsch [38] for solving the acoustic inverse scattering problem for soundsoft obstacles. The whole approach requires the Fréchet differentiability analysis of the far-field pattern of the solution to the scattering problem with respect to any parametrizations of the unknown boundary and, if it is possible, a characterization of the Fréchet derivative as a solution to a new elliptic boundary value problem. This was achieved by Kirsch using variational methods and alternative proofs were given by Potthast using the integral representation of the boundary to far-field operator and the material derivatives of the boundary integral operators [49] and by Kress and Päivarinta using a farfield identity [42]. We refer to [29, Chapter 4] for a review of existing iterative algorithms which lead to different choices of penalty terms in the framework of Hilbert spaces. An extension of these algorithms to Banach spaces can be found in [37] and references therein. A rigorous inverse algorithm based on regularized Gauß-Newton [5, 28] iterations and boundary integral equation formulations is described and analyzed by Hohage in [29, Chapter 4]. A fast and accurate reconstruction of the scattering object illuminated by a finite number of (or even one) incident plane waves is obtained [29, 23]. However a good initial guess and its approximate location have to be known from the beginning. The method has then been extended to other boundary conditions in acoustic scattering [31] since the needed theoretical results on the Fréchet derivatives where known [24, 25]. Second degree method for solving inverse obstacle scattering problems [27] are less popular since the characterizations of higher order Fréchet derivatives are rather difficult to obtain. Topological optimization consists in minimizing the nonlinear least square (or objective functional) by modifying the topology of the medium, as for example by creating multiple simply connected obstacles. Topological optimization method are very efficient for finding the number, the location and the size of the obstacles from a very small amount of data and without using any a priori initial information. We refer to [8] for a description of an iterative algorithm entirely based on topological derivatives for the identification problem of sound soft obstacles. Such method could be used as a starting point for determining intial guesses in the above mentioned geometric optimization algorithm. Nonlinear optimization methods have the additional interesting feature that one can combine them, through slight modifications in the algorithm, with gradient-based method to approximate the parameters of the obstacles and recover the boundary conditions satisfied by the scattered field at the interfaces.

Characterization of the Fréchet derivatives have been recently derived in electromagnetism for various boundary conditions $[41,21,16,45,50]$ and a fast inverse iterative algorithm is proposed in [30] for the identification of dielectric inclusions in unbounded homogeneous media. In elastodynamics, the characterization of the first Fréchet derivatives for the Dirichlet boundary condition case was obtained by Charalambopoulos in [9] extending Potthast's approach based on a boundary integral representation of the solution. An alternative proof and the Neumann boundary condition case have been recently investigated by the author in [45] extending Kress and Päivarinta' s approach based on a farfield identity. The purpose of this paper is to consider the case of transmission boundary conditions and to present a numerical solution method for iteratively recovering the shape of isotropic elastic inclusions.

The paper is organised as follows: In Section 2 we introduce the transmission problem of time-harmonic elastic waves across the smooth boundary of a three-dimensional bounded obstacle. The forward problem is reduced to systems of boundary integral equations following the very well-known direct and indirect methods initially developed in acoustics [40,43]. The nature of elastic waves makes rather difficult the construction of uniquely solvable weakly singular boundary integral formulation for the transmission problem. Contrary to the acoustic case, the resulting systems consist of strongly singular and hypersingular integral equations but we prove that they are uniquely solvable for the range of wavenumbers characterizing elastodynamic waves. Using these results, the far-field pattern of the solution to the transmission problem is given in terms of products of boundary integral operators and their inverses. Among all the existing methods above mentioned, we prove in Section 3 the Fréchet differentiability of the boundary to far-field operator using the recent results on the material derivatives of boundary integral operators with either a weakly, strongly or hypersingular kernel established in 
[15]. We give a characterization of the first Fréchet derivative and the adjoint operator, following ideas of [29], which is needed in the implementation of the iteratively regularized Gauß-Newton (IRGN) method in the framework of Hilbert spaces. In Section 4, we present the inverse scattering algorithm and we show numerical experiments in Section 5. The boundary integral equation systems are numerically solved by applying the high order spectral algorithm proposed by the author in [46] for solving elastodynamic problems in exterior domains. Similar convergence rates than those reported in [46] and $[19,20]$ are observed. The characterizations of the Fréchet derivatives are numerically compared to the finite difference method. The inverse scattering algorithm is applied to the shape reconstruction problem of convex and non convex star-shaped obstacles.

\section{$2 \quad$ Elastic scattering by penetrable obstacles}

Let $\Omega \subset \mathbb{R}^{3}$ be a bounded domain with a smooth closed orientable boundary $\Gamma$ of class $\mathscr{C}^{2}$ at least and outward unit normal vector $\boldsymbol{n}$ and let $\Omega^{c}$ denote the exterior domain $\mathbb{R}^{3} \backslash \bar{\Omega}$. Throughout the paper we denote by $H^{s}(\Omega)$, $H_{l o c}^{s}\left(\overline{\Omega^{c}}\right)$ and $H^{s}(\Gamma)$ the standard (local in the case of the exterior domain) complex valued, Hilbertian Sobolev space of order $s \in \mathbb{R}$ defined on $\overline{\Omega^{c}}$ and $\Gamma$ respectively (with the convention $H^{0}=L^{2}$.) Spaces of vector functions will be denoted by boldface letters, thus $\boldsymbol{H}^{s}=\left(H^{s}\right)^{3}$. We also use the surface differential operators: The tangential gradient $\nabla_{\Gamma}$, the surface divergence $\operatorname{div}_{\Gamma}$, the surface scalar curl curl ${ }_{\Gamma}$, the tangential vector curl $\operatorname{curl}_{\Gamma}$ and the scalar Laplace-Beltrami operator $\Delta_{\Gamma}$. For their definitions we refer to [47, pages 68-75].

For the formulation of the transmission problem we follow the notations of [11] and quote some important results on potential theory from [17] and [44, Chapter 3]. The propagation of time-harmonic elastic waves in the three-dimensional isotropic and homogeneous elastic medium is described by the Navier equation

$$
\Delta^{*} \boldsymbol{u}+\rho \omega^{2} \boldsymbol{u}=\mu \Delta \boldsymbol{u}+(\lambda+\mu) \nabla \operatorname{div} \boldsymbol{u}+\rho \omega^{2} \boldsymbol{u}=0,
$$

where $\omega>0$ is a fixed frequency. We assume that the Lamé parameters $\mu$ and $\lambda$ and the density $\rho$ take constant and different real values in $\Omega$ and $\Omega^{c}$. Moreover we assume $\mu>0$ and $3 \lambda+2 \mu>0$. We introduce the dimensionless Poisson's ratio $\nu=\frac{\lambda}{2(\lambda+\mu)}$. Then, we have $\frac{\mu}{\lambda+2 \mu}=\frac{1-2 \nu}{2(1-\nu)}, \frac{\lambda+\mu}{\lambda+2 \mu}=\frac{1}{2(1-\nu)}$ and $\frac{\lambda+3 \mu}{\lambda+2 \mu}=\frac{3-4 \nu}{2(1-\nu)}$. We set

$$
\begin{array}{ll}
\boldsymbol{H}^{1}\left(\Omega, \Delta^{*}\right) & :=\left\{\boldsymbol{u} \in \boldsymbol{H}^{1}(\Omega): \Delta^{*} \boldsymbol{u} \in \boldsymbol{L}^{2}(\Omega)\right\} \\
\boldsymbol{H}_{l o c}^{1}\left(\Omega^{c}, \Delta^{*}\right) & :=\left\{\boldsymbol{u} \in \boldsymbol{H}_{l o c}^{1}\left(\overline{\Omega^{c}}\right): \Delta^{*} \boldsymbol{u} \in \boldsymbol{L}_{l o c}^{2}\left(\overline{\Omega^{c}}\right)\right\} .
\end{array}
$$

We use the following traces :

$$
\begin{aligned}
\frac{\partial}{\partial \boldsymbol{n}} & =\boldsymbol{n} \cdot \nabla,(\text { normal derivative }) \\
\boldsymbol{T}(\boldsymbol{n}, \partial) & =2 \mu \frac{\partial}{\partial \boldsymbol{n}}+\lambda \boldsymbol{n} \operatorname{div}+\mu \boldsymbol{n} \times \mathbf{c u r l},(\text { traction trace }) .
\end{aligned}
$$

We note that, due to the trace lemma, $\boldsymbol{u}_{\mid \Gamma} \in \boldsymbol{H}^{\frac{1}{2}}(\Gamma)$ for $\boldsymbol{u} \in \boldsymbol{H}^{1}\left(\Omega, \Delta^{*}\right) \cup \boldsymbol{H}_{l o c}^{1}\left(\Omega^{c}, \Delta^{*}\right)$. The normal derivative $\frac{\partial}{\partial \boldsymbol{n}} \boldsymbol{u}_{\mid \Gamma}$ and the traction derivative $\boldsymbol{T} \boldsymbol{u}_{\mid \Gamma}$ are both defined as distributions in $\boldsymbol{H}^{-\frac{1}{2}}(\Gamma)$.

In what follows, we will use a lower or upper index $i$ for all quantities related to the penetrable scatterer $\Omega$ and a lower or upper index $e$ for all quantities related to the exterior domain $\Omega^{c}$. The forward problem is formulated as follows: Given vector densities $\boldsymbol{f} \in \boldsymbol{H}^{\frac{1}{2}}(\Gamma)$ and $\boldsymbol{g} \in \boldsymbol{H}^{-\frac{1}{2}}(\Gamma)$, find the solution $\left(\boldsymbol{u}^{i}, \boldsymbol{u}^{e}\right) \in$ $\boldsymbol{H}^{1}\left(\Omega, \Delta_{i}^{*}\right) \times \boldsymbol{H}_{l o c}^{1}\left(\Omega^{c}, \Delta_{e}^{*}\right)$ to the system of Navier equations in $\Omega \cup \Omega^{c}$

$$
\begin{aligned}
\Delta_{i}^{*} \boldsymbol{u}^{i}+\rho_{i} \omega^{2} \boldsymbol{u}^{i}=0 & \text { in } \Omega, \\
\Delta_{e}^{*} \boldsymbol{u}^{e}+\rho_{e} \omega^{2} \boldsymbol{u}^{e}=0 & \text { in } \Omega^{c},
\end{aligned}
$$

which satisfies the transmission boundary conditions

$$
\boldsymbol{u}^{i}=\boldsymbol{u}^{e}+\boldsymbol{f} \quad \text { on } \Gamma
$$


and

$$
\boldsymbol{T}_{i} \boldsymbol{u}^{i}=\boldsymbol{T}_{e} \boldsymbol{u}^{e}+\boldsymbol{g} \quad \text { on } \Gamma .
$$

In addition the scattered field $\boldsymbol{u}^{e}$ has to satisfy the Kupradze radiation condition

$$
\lim _{r \rightarrow \infty} r\left(\frac{\partial \boldsymbol{u}_{p}^{e}}{\partial r}-i \kappa_{p}^{e} \boldsymbol{u}_{p}^{e}\right)=0, \quad \lim _{r \rightarrow \infty} r\left(\frac{\partial \boldsymbol{u}_{s}^{e}}{\partial r}-i \kappa_{s}^{e} \boldsymbol{u}_{s}^{e}\right)=0, \quad r=|\boldsymbol{x}|,
$$

uniformly in all directions. Here, the longitudinal wave is given by $\boldsymbol{u}_{p}^{e}=-\left(\kappa_{p}^{e}\right)^{-2} \boldsymbol{\nabla} \operatorname{div} \boldsymbol{u}^{e}$ and the transversal wave is given by $\boldsymbol{u}_{s}^{e}=\boldsymbol{u}^{e}-\boldsymbol{u}_{p}^{e}$ associated with the respective exterior wavenumbers $\kappa_{p}^{e}$ and $\kappa_{s}^{e}$ given by $\kappa_{p}^{a}=\omega \sqrt{\rho_{a}\left(\lambda_{a}+2 \mu_{a}\right)^{-1}}$ and $\kappa_{s}^{a}=\omega \sqrt{\rho_{a} \mu_{a}^{-1}}$ for $a=i, e$. We point out that the Kupradze radiation conditions are sufficient conditions [44, Theorem 2.9, pp. 127] to obtain the following useful radiation conditions

$$
\lim _{r \rightarrow \infty} r\left(T_{e} \boldsymbol{u}_{p}^{e}-i \kappa_{p}^{e}\left(\lambda_{e}+2 \mu_{e}\right) \boldsymbol{u}_{p}^{e}\right)=0, \quad \lim _{r \rightarrow \infty} r\left(T_{e} \boldsymbol{u}_{s}^{e}-i \kappa_{s}^{e} \mu_{e} \boldsymbol{u}_{s}^{e}\right)=0, \quad r=|\boldsymbol{x}|,
$$

The conditions (2.2) together with the first Green formula for the Navier equation [17, Lemma 2.1] and Rellich's Lemma allow us to prove that the homogeneous transmission problem admits at most one solution. Existence of a solution can be proved using boundary integral equation methods. Following [40, 43], we give an alternative proof to [17] below, but available for smooth boundaries only.

Setting $G(\kappa, \boldsymbol{z})=\frac{e^{i \kappa|\boldsymbol{z}|}}{4 \pi|\boldsymbol{z}|}$, the fundamental solution of the Navier equation is given by

$$
\Phi^{a}(\boldsymbol{x}, \boldsymbol{y})=\frac{1}{\mu_{a}}\left(G\left(\kappa_{s}^{a}, \boldsymbol{x}-\boldsymbol{y}\right) \mathrm{I}_{\mathbb{R}^{3}}+\frac{1}{\left(\kappa_{s}^{a}\right)^{2}} \nabla_{\boldsymbol{x}}^{\top} \boldsymbol{\nabla}_{\boldsymbol{x}}\left(G\left(\kappa_{s}^{a}, \boldsymbol{x}-\boldsymbol{y}\right)-G\left(\kappa_{p}^{a}, \boldsymbol{x}-\boldsymbol{y}\right)\right)\right) .
$$

It is a $3 \times 3$ matrix-valued function and we have $\Phi^{a}(\boldsymbol{x}, \boldsymbol{y})={ }^{\top} \Phi^{a}(\boldsymbol{x}, \boldsymbol{y})=\Phi^{a}(\boldsymbol{y}, \boldsymbol{x})$. For a solution to the Navier equation (2.1b), one can derive the Somigliana integral representation formula for $\boldsymbol{x} \in \Omega$

$$
\boldsymbol{u}^{i}(\boldsymbol{x})=\int_{\Gamma}\left(\Phi^{i}(\boldsymbol{x}, \boldsymbol{y}) \boldsymbol{T}_{i, \boldsymbol{y}} \boldsymbol{u}^{i}(\boldsymbol{y})-{ }^{\mathrm{\top}}\left[\boldsymbol{T}_{i, \boldsymbol{y}} \Phi^{i}(\boldsymbol{x}, \boldsymbol{y})\right] \boldsymbol{u}^{i}(\boldsymbol{y})\right) d s(\boldsymbol{y}),
$$

where $\boldsymbol{T}_{\boldsymbol{y}}=\boldsymbol{T}\left(\boldsymbol{n}(\boldsymbol{y}), \partial_{\boldsymbol{y}}\right)$ and $\boldsymbol{T}_{\boldsymbol{y}} \Phi(\boldsymbol{x}, \boldsymbol{y})$ is the tensor obtained by applying the traction operator $\boldsymbol{T}_{\boldsymbol{y}}$ to each column of $\Phi(\boldsymbol{x}, \boldsymbol{y})$. For a solution to the Navier equation (2.1b) that satisfies the Kupraze radiation condition, one can derive the Somigliana integral representation formula for $\boldsymbol{x} \in \Omega^{c}$ :

$$
\boldsymbol{u}^{e}(\boldsymbol{x})=\int_{\Gamma}\left({ }^{\top}\left[\boldsymbol{T}_{e, \boldsymbol{y}} \Phi^{e}(\boldsymbol{x}, \boldsymbol{y})\right] \boldsymbol{u}^{e}(\boldsymbol{y})-\Phi^{e}(\boldsymbol{x}, \boldsymbol{y}) \boldsymbol{T}_{e, \boldsymbol{y}} \boldsymbol{u}^{e}(\boldsymbol{y})\right) d s(\boldsymbol{y}) .
$$

The transmission problem of time-harmonic elastic waves by a bounded obstacle $\Omega$ can be reduced in several different ways to a system of uniquely solvable boundary integral equations. We present two different approaches. The Calderón projectors for the time-harmonic Navier equation are

$$
P_{a}^{ \pm}=\left(\begin{array}{cc} 
\pm \frac{1}{2} \mathrm{I}+D_{a} & -S_{a} \\
N_{a} & \pm \frac{1}{2} \mathrm{I}-D_{a}^{\prime}
\end{array}\right) .
$$

where I is the identity operator and the boundary integral operators are defined by

$$
\begin{aligned}
S_{a} \boldsymbol{\varphi}(\boldsymbol{x}) & =\int_{\Gamma} \Phi^{a}(\boldsymbol{x}, \boldsymbol{y}) \boldsymbol{\varphi}(\boldsymbol{y}) d s(\boldsymbol{y}), \\
D_{a} \boldsymbol{\psi}(\boldsymbol{x}) & =\int_{\Gamma}^{\mathrm{T}_{T}}\left[\boldsymbol{T}_{a, \boldsymbol{y}} \Phi^{a}(\boldsymbol{x}, \boldsymbol{y})\right] \boldsymbol{\psi}(\boldsymbol{y}) d s(\boldsymbol{y}), \\
D_{a}^{\prime} \boldsymbol{\varphi}(\boldsymbol{x}) & =\int_{\Gamma} \boldsymbol{T}_{a, \boldsymbol{x}}\left\{\Phi^{a}(\boldsymbol{x}, \boldsymbol{y}) \boldsymbol{\varphi}(\boldsymbol{y})\right\} d s(\boldsymbol{y}), \\
N_{a} \boldsymbol{\psi}(\boldsymbol{x}) & =\int_{\Gamma} \boldsymbol{T}_{a, \boldsymbol{x}}\left\{{ }^{\mathrm{T}}\left[\boldsymbol{T}_{a, \boldsymbol{y}} \Phi^{a}(\boldsymbol{x}, \boldsymbol{y})\right] \boldsymbol{\psi}(\boldsymbol{y})\right\} d s(\boldsymbol{y}) .
\end{aligned}
$$


The operator $S_{a}$ is bounded from $\boldsymbol{H}^{-\frac{1}{2}}(\Gamma)$ to $\boldsymbol{H}^{\frac{1}{2}}(\Gamma)$ and compact from $\boldsymbol{H}^{-\frac{1}{2}}(\Gamma)$ to itself. The operators $D_{a}: \boldsymbol{H}^{\frac{1}{2}}(\Gamma) \rightarrow \boldsymbol{H}^{\frac{1}{2}}(\Gamma)$ and $D_{a}^{\prime}: \boldsymbol{H}^{-\frac{1}{2}}(\Gamma) \rightarrow \boldsymbol{H}^{-\frac{1}{2}}(\Gamma)$ are bounded and have a strongly singular kernel. The operator $N_{a}: \boldsymbol{H}^{\frac{1}{2}}(\Gamma) \rightarrow \boldsymbol{H}^{-\frac{1}{2}}(\Gamma)$ is bounded and has a hypersingular kernel.

For $a=i, e$, we set $\boldsymbol{p}_{a}={ }^{\top}\left(\boldsymbol{u}_{\mid \Gamma}^{a}, \boldsymbol{T}_{a} \boldsymbol{u}_{\mid \Gamma}^{a}\right)$. We have the following results

$$
P_{e}^{+} \boldsymbol{p}_{e}=\boldsymbol{p}_{e}, \quad P_{e}^{-} \boldsymbol{p}_{e}=\mathbf{0}, \quad P_{i}^{+} \boldsymbol{p}_{i}=\mathbf{0}, \quad P_{i}^{-} \boldsymbol{p}_{i}=-\boldsymbol{p}_{i} .
$$

The direct approach [40, Section 4.2] is used when $\boldsymbol{f}$ and $\boldsymbol{g}$ are the boundary data of a time-harmonic incident elastic wave $\boldsymbol{u}^{i n c}$ which is assumed to solve the Navier equation in the absence of any scatterer. In this case, we have

$$
P_{e}^{+} \boldsymbol{p}_{\text {inc }}=\mathbf{0} \text { and } P_{e}^{-} \boldsymbol{p}_{\text {inc }}=-\boldsymbol{p}_{\text {inc }} \text { with } \boldsymbol{p}_{\text {inc }}=\left(\begin{array}{c}
\boldsymbol{u}_{\mid \Gamma}^{i n c} \\
\boldsymbol{T}_{e} \boldsymbol{u}_{\mid \Gamma}^{i n c}
\end{array}\right) .
$$

Using these results, we derive the following system of boundary integral equations of unknown $\boldsymbol{p}=\boldsymbol{p}_{i}=\boldsymbol{p}_{e}+\boldsymbol{p}_{\text {inc }}$

$$
\left(P_{i}^{+}-P_{e}^{-}\right) \boldsymbol{p}=\left(\begin{array}{cc}
\mathrm{I}+\left(D_{i}-D_{e}\right) & -\left(S_{i}-S_{e}\right) \\
N_{i}-N_{e} & \mathrm{I}-\left(D_{i}^{\prime}-D_{e}^{\prime}\right)
\end{array}\right) \boldsymbol{p}=\boldsymbol{p}_{\text {inc }} .
$$

If one solves the boundary integral equation system (2.5) then the solution of the transmission problem is given by $(2.3)$ and

$$
\boldsymbol{u}^{e}(\boldsymbol{x})=\int_{\Gamma}\left({ }^{\top}\left[\boldsymbol{T}_{e, \boldsymbol{y}} \Phi^{e}(\boldsymbol{x}, \boldsymbol{y})\right]\left(\boldsymbol{u}^{e}+\boldsymbol{u}^{i n c}\right)(\boldsymbol{y})-\Phi^{e}(\boldsymbol{x}, \boldsymbol{y}) \boldsymbol{T}_{e, \boldsymbol{y}}\left(\boldsymbol{u}^{e}+\boldsymbol{u}^{i n c}\right)(\boldsymbol{y})\right) d s(\boldsymbol{y})
$$

The indirect approach [40, Section 4.2] or [43] can be used for all boundary data $(\boldsymbol{f}, \boldsymbol{g}) \in \boldsymbol{H}^{\frac{1}{2}}(\Gamma) \times \boldsymbol{H}^{-\frac{1}{2}}(\Gamma)$. It is based on the layer ansatz

$$
\begin{array}{rll}
\boldsymbol{u}_{i}(\boldsymbol{x}) & =\int_{\Gamma}^{\mathrm{T}}\left[\boldsymbol{T}_{i, \boldsymbol{y}} \Phi^{i}(\boldsymbol{x}, \boldsymbol{y})\right] \boldsymbol{\psi}(\boldsymbol{y}) d s(\boldsymbol{y})+\int_{\Gamma} \Phi^{i}(\boldsymbol{x}, \boldsymbol{y}) \boldsymbol{\varphi}(\boldsymbol{y}) d s(\boldsymbol{y}), & \boldsymbol{x} \in \Omega \\
\boldsymbol{u}_{e}(\boldsymbol{x}) & =\int_{\Gamma}^{\mathrm{T}}\left[\boldsymbol{T}_{e, \boldsymbol{y}} \Phi^{e}(\boldsymbol{x}, \boldsymbol{y})\right] \boldsymbol{\psi}(\boldsymbol{y}) d s(\boldsymbol{y})+\int_{\Gamma} \Phi^{e}(\boldsymbol{x}, \boldsymbol{y}) \boldsymbol{\varphi}(\boldsymbol{y}) d s(\boldsymbol{y}), & \boldsymbol{x} \in \Omega^{c} .
\end{array}
$$

We set $\boldsymbol{p}={ }^{\top}(\boldsymbol{\psi}, \boldsymbol{\varphi})$ and $\tilde{\boldsymbol{p}}={ }^{\mathrm{T}}(\boldsymbol{\psi},-\boldsymbol{\varphi})$, then $\boldsymbol{p}_{e}=P_{e}^{+} \tilde{\boldsymbol{p}}$ and $\boldsymbol{p}_{i}=P_{i}^{-} \tilde{\boldsymbol{p}}$. We obtain the following system of boundary integral equations

$$
\left(\begin{array}{cc}
\mathrm{I} & 0 \\
0 & -\mathrm{I}
\end{array}\right)\left(P_{e}^{+}-P_{i}^{-}\right) \tilde{\boldsymbol{p}}=\left[\left(\begin{array}{cc}
\mathrm{I}-\left(D_{i}-D_{e}\right) & -\left(S_{i}-S_{e}\right) \\
N_{i}-N_{e} & \mathrm{I}+\left(D_{i}^{\prime}-D_{e}^{\prime}\right)
\end{array}\right)\right] \boldsymbol{p}=\left(\begin{array}{c}
-\boldsymbol{f} \\
\boldsymbol{g}
\end{array}\right) .
$$

If one solves the boundary integral equation system (2.9) then the solution of the transmission problem is given by (2.7) and (2.8). In the sequel, we denote by $\mathbf{I}_{o p}$ the boundary integral equation operator in (2.9) and by $\mathbf{I}_{o p}^{\dagger}$ the one in $(2.5)$.

Theorem 2.1 Let $(\boldsymbol{f}, \boldsymbol{g}) \in \boldsymbol{H}^{\frac{1}{2}}(\Gamma) \times \boldsymbol{H}^{-\frac{1}{2}}(\Gamma)$. The boundary integral equation system (2.9) admits one and only one solution $\boldsymbol{p}={ }^{\top}(\boldsymbol{\psi}, \boldsymbol{\varphi}) \in \boldsymbol{H}^{\frac{1}{2}}(\Gamma) \times \boldsymbol{H}^{-\frac{1}{2}}(\Gamma)$.

Proof. We proceed in two steps. First we prove injectivity following [43, proof (ii) of Theorem 4.2], then we prove that the boundary integral equation operator is a Fredholm operator of index zero. We conclude using Riesz theory.

- Let consider the homogeneous form of the system (2.9) which means $\boldsymbol{f}=\mathbf{0}$ and $\boldsymbol{g}=\mathbf{0}$. Since the homogeneous form of the transmission problem (2.1a)-(2.1e) admits the trivial solution only, we obtain $\boldsymbol{u}_{e}=\mathbf{0}$ in $\Omega^{c}$ and $\boldsymbol{u}^{i}=\mathbf{0}$ in $\Omega$. It follows $P_{e}^{+} \tilde{\boldsymbol{p}}=\mathbf{0}$ and $P_{i}^{-} \tilde{\boldsymbol{p}}=\mathbf{0}$ and

$$
P_{e}^{-} \tilde{\boldsymbol{p}}=-\tilde{\boldsymbol{p}} \quad \text { and } P_{i}^{+} \tilde{\boldsymbol{p}}=\tilde{\boldsymbol{p}}
$$


Now we introduce the following displacement fields

$$
\begin{aligned}
\boldsymbol{v}^{i}(\boldsymbol{x}) & =\int_{\Gamma}^{\mathrm{T}}\left[\boldsymbol{T}_{i, \boldsymbol{y}} \Phi^{i}(\boldsymbol{x}, \boldsymbol{y})\right] \psi(\boldsymbol{y}) d s(\boldsymbol{y})+\int_{\Gamma} \Phi^{i}(\boldsymbol{x}, \boldsymbol{y}) \boldsymbol{\varphi}(\boldsymbol{y}) d s(\boldsymbol{y}), \quad \boldsymbol{x} \in \Omega^{c} \\
\boldsymbol{v}^{e}(\boldsymbol{x}) & =-\int_{\Gamma}^{\mathrm{T}}\left[\boldsymbol{T}_{e, \boldsymbol{y}} \Phi^{e}(\boldsymbol{x}, \boldsymbol{y})\right] \boldsymbol{\psi}(\boldsymbol{y}) d s(\boldsymbol{y})-\int_{\Gamma} \Phi^{e}(\boldsymbol{x}, \boldsymbol{y}) \boldsymbol{\varphi}(\boldsymbol{y}) d s(\boldsymbol{y}), \quad \boldsymbol{x} \in \Omega .
\end{aligned}
$$

We deduce ${ }^{\top}\left(\boldsymbol{v}_{\mid \Gamma}^{e}, \boldsymbol{T}_{e} \boldsymbol{v}_{\mid \Gamma}^{e}\right)=-P_{e}^{-} \tilde{\boldsymbol{p}}$ and ${ }^{\mathrm{T}}\left(\boldsymbol{v}_{\mid \Gamma}^{i}, \boldsymbol{T}_{i} \boldsymbol{v}_{\mid \Gamma}^{i}\right)=P_{i}^{+} \tilde{\boldsymbol{p}}$. From (2.10), we deduce $\left(\boldsymbol{v}_{e}, \boldsymbol{v}_{i}\right)$ solves the homogeneous form of the transmission problem (2.1a)-(2.1e) where we have interchanged the interior parameters $\mu_{i}, \lambda_{i}, \rho_{i}$ with the exterior parameters $\mu_{e}, \lambda_{e}, \rho_{e}$. Hence, the solution $\left(\boldsymbol{v}^{e}, \boldsymbol{v}^{i}\right) \in \boldsymbol{H}^{1}\left(\Omega, \Delta_{e}^{*}\right) \times$ $\boldsymbol{H}_{l o c}^{1}\left(\Omega^{c}, \Delta_{i}^{*}\right)$ is identically equal to zero and coming back to $(2.10)$ we obtain $\tilde{\boldsymbol{p}}=\mathbf{0}=\boldsymbol{p}$.

- Now we prove that the boundary integral equation operator is a compact perturbation of an invertible operator. To this end we consider the principal parts of the boundary integral operators obtained in [18]. To describe their behavior we use an orthonormal basis $\left(\left(\mathcal{Y}_{j}^{(0)}\right)_{0 \leq j<N_{0}},\left(\mathcal{Y}_{j}^{(1)}\right)_{j \in \mathbb{N}^{*}},\left(\mathcal{Y}_{j}^{(2)}\right)_{j \in \mathbb{N}^{*}},\left(\mathcal{Y}_{j}^{(3)}\right)_{j \in \mathbb{N}}\right)$ of $\boldsymbol{L}^{2}(\Gamma)$ consisting of the eigenfunctions of the scalar and vector Laplace-Beltrami operators. (It seems easier than the use of the Fourier analysis especially in linear elasticity [2].) We precise that $\left(\mathcal{Y}_{j}^{(0)}\right)_{0 \leq j<N_{0}}$ span the nullspace $\mathcal{N}$ of the vector Laplace-Beltrami operator and $\left(\left(\mathcal{Y}_{j}^{(1)}\right)_{j \in \mathbb{N}^{*}},\left(\mathcal{Y}_{j}^{(2)}\right)_{j \in \mathbb{N}^{*}}\right)$ span the set of tangential densities with non vanishing surface divergence or surface curl. We refer to the appendix for more details. From [18, Lemmas 3.2 to 3.5] we deduce that the principal parts of the boundary integral operators, written in the function basis $\left(\mathcal{Y}_{j}^{(1)}, \mathcal{Y}_{j}^{(2)}, \mathcal{Y}_{j}^{(3)}\right)$, behaves as follows when $j \rightarrow \infty$

$$
\begin{gathered}
P_{-1}\left(S_{a}\right)=\left(\begin{array}{ccc}
\frac{3-4 \nu_{a}}{8 \mu_{a}\left(1-\nu_{a}\right)} \beta_{j}^{-\frac{1}{2}} & 0 & 0 \\
0 & \frac{1}{2 \mu_{a}} \beta_{j}^{-\frac{1}{2}} & 0 \\
0 & 0 & \frac{3-4 \nu_{a}}{8 \mu_{a}\left(1-\nu_{a}\right)} \beta_{j}^{-\frac{1}{2}}
\end{array}\right)+O\left(\beta_{j}^{-1}\right) \\
P_{0}\left(D_{a}\right)=\left(\begin{array}{ccc}
0 & 0 & \frac{1-2 \nu_{a}}{4\left(1-\nu_{a}\right)} \\
0 & 0 & 0 \\
\frac{1-2 \nu_{a}}{4\left(1-\nu_{a}\right)} & 0 & 0
\end{array}\right)+O\left(\beta_{j}^{-\frac{1}{2}}\right), P_{0}\left(D_{a}^{\prime}\right)={ }^{\top} P_{0}\left(D_{a}\right)
\end{gathered}
$$

and

$$
P_{1}\left(N_{a}\right)=\left(\begin{array}{ccc}
-\frac{\mu_{a}}{2\left(1-\nu_{a}\right)} \beta_{j}^{\frac{1}{2}} & 0 & 0 \\
0 & -\frac{\mu_{a}}{2} \beta_{j}^{\frac{1}{2}} & 0 \\
0 & 0 & -\frac{\mu_{a}}{2\left(1-\nu_{a}\right)} \beta_{j}^{\frac{1}{2}}
\end{array}\right)+O(1) .
$$

Let $j_{\boldsymbol{L}^{2} \rightarrow \boldsymbol{H}^{s}}$ be the isomorphism between $\boldsymbol{L}^{2}(\Gamma)$ and $\boldsymbol{H}^{s}(\Gamma)$ defined by (A.2). We extend this definition to its bevariate analogue $j_{\boldsymbol{L}^{2} \times \boldsymbol{L}^{2} \rightarrow \boldsymbol{H}^{s} \times \boldsymbol{H}^{t}(\Gamma)}(\boldsymbol{\varphi}, \boldsymbol{\psi})=\left(j_{\boldsymbol{L}^{2} \rightarrow \boldsymbol{H}^{s}}(\boldsymbol{\varphi}), j_{\boldsymbol{L}^{2} \rightarrow \boldsymbol{H}^{t}}(\boldsymbol{\psi})\right)$. To homogenize the units we use the following invertible linear transform : $\mathbf{U}:(\boldsymbol{\varphi}, \boldsymbol{\psi}) \mapsto\left(\boldsymbol{\varphi}, \mu_{e} \boldsymbol{\psi}\right)$. By composition, the operator $\mathbf{I}_{o p}$ is a Fredholm operator of index zero on $\boldsymbol{H}^{\frac{1}{2}}(\Gamma) \times \boldsymbol{H}^{-\frac{1}{2}}(\Gamma)$ if and only if

$$
\mathbf{J}_{o p}=j_{\boldsymbol{H}^{\frac{1}{2}} \times \boldsymbol{H}^{-\frac{1}{2}} \rightarrow \boldsymbol{L}^{2} \times \boldsymbol{L}^{2}} \circ \mathbf{U}^{-1} \circ \mathbf{I}_{o p} \circ \mathbf{U} \circ j_{\boldsymbol{L}^{2} \times \boldsymbol{L}^{2} \rightarrow \boldsymbol{H}^{\frac{1}{2}} \times \boldsymbol{H}^{-\frac{1}{2}}}
$$

is a Fredholm operator of index zero on $\boldsymbol{L}^{2}(\Gamma) \times \boldsymbol{L}^{2}(\Gamma)$. Now we denote by $\left(\underline{\varphi_{j}^{(0)}}\right)_{0 \leq j<N_{0}},\left(\underline{\varphi_{j}^{(1)}}\right)_{j \geq 1}$, $\left.\underline{\left(\varphi_{j}^{(2)}\right.}\right)_{j \geq 1}$ and $\left(\underline{\varphi_{j}^{(3)}}\right)_{j \geq 0}$ the Fourier coefficients of the density $\boldsymbol{\varphi} \in \boldsymbol{L}^{2}(\Gamma)$ and by $\left.\overline{\left(\psi_{j}^{(0)}\right.}\right)_{0 \leq j<N_{0}}, \underline{\left(\overline{\psi_{j}^{(1)}}\right)_{j \geq 1}}$, 
$\left(\underline{\psi_{j}^{(2)}}\right)_{j \geq 1}$ and $\left(\psi_{j}^{(3)}\right)_{j \geq 0}$ the Fourier coefficients of the density $\boldsymbol{\psi} \in \boldsymbol{L}^{2}(\Gamma)$. The principal part of $\mathbf{J}_{o p}$ is a compact perturbation of an invertible operator $\mathbf{A}$ that can be rewritten as a sequence of 2 systems with respectively 2 equations for the 2 unknowns $\left(\varphi_{j}^{(2)}, \psi_{j}^{(2)}\right)$ and with 4 equations for the 4 unknwons $\underline{\left(\varphi_{j}^{(1)}\right.}, \underline{\varphi_{j}^{(3)}}, \underline{\psi_{j}^{(1)}}, \underline{\left.\psi_{j}^{(3)}\right)}$. The associated matrices are

$$
\left(\begin{array}{cc}
1 & -\frac{1}{2}\left(\frac{\mu_{e}}{\mu_{i}}-1\right) \\
-\frac{1}{2}\left(\frac{\mu_{i}}{\mu_{e}}-1\right) & 1
\end{array}\right)
$$

whose determinant is equal to $\frac{1}{2}+\frac{1}{4}\left(\frac{\mu_{i}}{\mu_{e}}+\frac{\mu_{e}}{\mu_{i}}\right) \neq 0$ and

$$
\left(\begin{array}{cccc}
1 & a & b & 0 \\
a & 1 & 0 & b \\
c & 0 & 1 & -a \\
0 & c & -a & 1
\end{array}\right) \text { with }\left\{\begin{aligned}
a & =\frac{1-2 \nu_{e}}{4\left(1-\nu_{e}\right)}-\frac{1-2 \nu_{i}}{4\left(1-\nu_{i}\right)} \\
b & =\left(\frac{3-4 \nu_{e}}{8\left(1-\nu_{e}\right)}-\frac{\mu_{e}}{\mu_{i}} \frac{3-4 \nu_{i}}{8\left(1-\nu_{i}\right)}\right) \\
c & =\left(\frac{1}{2\left(1-\nu_{e}\right)}-\frac{\mu_{i}}{\mu_{e}} \frac{1}{2\left(1-\nu_{i}\right)}\right)
\end{aligned}\right.
$$

whose determinant is equal to $\left(a^{2}+b c-1\right)^{2} \neq 0$. Indeed, under the hypothesis on the Lamé parameters we have $2\left(1-\nu_{a}\right)>\left(1-2 \nu_{a}\right)>0$ and $4\left(1-\nu_{a}\right)^{2}>\left(3-4 \nu_{a}\right)>0$. It follows $a^{2}<\frac{1}{2}-\frac{\left(1-2 \nu_{e}\right)\left(1-2 \nu_{i}\right)}{8\left(1-\nu_{e}\right)\left(1-\nu_{i}\right)}<\frac{1}{2}$, $b c<\frac{1}{2}-\frac{\mu_{e}}{\mu_{i}} \frac{3-4 \nu_{i}}{16\left(1-\nu_{i}\right)\left(1-\nu_{e}\right)}-\frac{\mu_{i}}{\mu_{e}} \frac{3-4 \nu_{e}}{16\left(1-\nu_{i}\right)\left(1-\nu_{e}\right)}<\frac{1}{2}$ and $1-a^{2}-b c>0$. We choose $\mathbf{A}$ as the identity operator on $\left(\mathcal{N} \oplus \mathbb{C} \mathcal{Y}_{0}^{(0)}\right)^{2}$. We have $\left(\mathbf{J}_{o p}-\mathbf{A}\right)=O\left(\beta_{j}^{-\frac{1}{2}}\right)$ in the function basis $\left(\boldsymbol{Y}_{j}^{(1)}, \mathcal{Y}_{j}^{(2)}, \mathcal{Y}_{j}^{(3)}\right)$ when $j \rightarrow \infty$. Then $\mathbf{J}_{o p}=\mathbf{A}+\left(\mathbf{J}_{o p}-\mathbf{A}\right)$ and $\left(\mathbf{J}_{o p}-\mathbf{A}\right)$ is compact from $\boldsymbol{L}^{2}(\Gamma) \times \boldsymbol{L}^{2}(\Gamma)$ to itself.

Remark 2.2 We also deduce the unique solvability of the equation (2.5) since $\mathbf{I}_{o p}^{\dagger}$ is related to the adjoint operator $\left(\mathbf{I}_{o p}\right)_{\mid \mathbf{L}^{2}}^{\mathrm{t}}$ of $\mathbf{I}_{\text {op }}$ for the $\boldsymbol{L}^{2}$ duality product that can be written as folows

$$
\left(\mathbf{I}_{o p}\right)_{\mathbf{L}^{2}}^{\mathrm{t}}=\left(\begin{array}{cc}
0 & \mathrm{I} \\
\mathrm{I} & 0
\end{array}\right) \mathbf{I}_{o p}^{\dagger}\left(\begin{array}{cc}
0 & \mathrm{I} \\
\mathrm{I} & 0
\end{array}\right) .
$$

An interesting feature of the systems (2.5) and (2.9) is that one only has to implement and store four boundary integral operators for both systems. Numerical experiments are presented in Tables 1 and 2.

The radiation condition implies that the scattered field has an asymptotic behavior of the form

$$
\boldsymbol{u}^{e}(\boldsymbol{x})=\frac{e^{i \kappa_{p}^{e}|\boldsymbol{x}|}}{|\boldsymbol{x}|} \boldsymbol{u}_{p}^{\infty}(\hat{\boldsymbol{x}})+\frac{e^{i \kappa_{s}^{e}|\boldsymbol{x}|}}{|\boldsymbol{x}|} \boldsymbol{u}_{s}^{\infty}(\hat{\boldsymbol{x}})+O\left(\frac{1}{|\boldsymbol{x}|}\right), \quad|\boldsymbol{x}| \rightarrow \infty,
$$

uniformly in all directions $\hat{\boldsymbol{x}}=\frac{\boldsymbol{x}}{|\boldsymbol{x}|}$. The fields $\boldsymbol{u}_{p}^{\infty}$ and $\boldsymbol{u}_{s}^{\infty}$ are defined on the unit sphere $\mathbb{S}^{2}$ in $\mathbb{R}^{3}$ and known as the longitudinal and the transversal far-field pattern, respectively. Using the direct approach (2.5) the far-field pattern can be computed via the integral representation formula

$$
\boldsymbol{u}^{\infty}=\left(\begin{array}{cc}
\mathscr{F}_{\mathrm{N}} & -\mathscr{F}_{\mathrm{D}}
\end{array}\right)\left(\begin{array}{c}
\boldsymbol{u}^{i} \\
\boldsymbol{T}_{i} \boldsymbol{u}^{i}
\end{array}\right)
$$

and using the indirect one (2.9) the far-field pattern can be computed via the integral representation formula

$$
\boldsymbol{u}^{\infty}=\left(\begin{array}{ll}
\mathscr{F}_{\mathrm{N}} & \mathscr{F}_{\mathrm{D}}
\end{array}\right)\left(\begin{array}{l}
\psi \\
\varphi
\end{array}\right)
$$


where the far-field operator $\mathscr{F}_{\mathrm{D}}$ is defined by (see [3, equations $(2.12)$ and $\left.(2.13)\right]$ )

$$
\mathscr{F}_{\mathrm{D}} \boldsymbol{\varphi}(\hat{\boldsymbol{x}})=\int_{\Gamma}\left(\frac{1}{\mu_{e}}\left[\mathrm{I}_{\mathbb{R}^{3}}-\hat{\boldsymbol{x}} \otimes \hat{\boldsymbol{x}}\right] \frac{e^{-i \kappa_{s}^{e} \hat{\boldsymbol{x}} \cdot \boldsymbol{y}}}{4 \pi}+\frac{1}{\lambda_{e}+2 \mu_{e}}[\hat{\boldsymbol{x}} \otimes \hat{\boldsymbol{x}}] \frac{e^{-i \kappa_{p}^{e} \hat{\boldsymbol{x}} \cdot \boldsymbol{y}}}{4 \pi}\right) \boldsymbol{\varphi}(\boldsymbol{y}) d s(\boldsymbol{y}) .
$$

and the far-field operator $\mathscr{F}_{\mathrm{N}}$ is defined by (see [3, equations $(2.12)$ and $\left.(2.13)\right]$ )

$$
\mathscr{F}_{\mathrm{N}} \boldsymbol{\psi}(\hat{\boldsymbol{x}})=\int_{\Gamma}\left(\frac{1}{\mu_{e}}{ }^{\mathrm{T}}\left[\boldsymbol{T}_{e, \boldsymbol{y}}\left[\mathrm{I}_{\mathbb{R}^{3}}-\hat{\boldsymbol{x}} \otimes \hat{\boldsymbol{x}}\right] \frac{e^{-i \kappa_{s}^{e} \hat{\boldsymbol{x}} \cdot y}}{4 \pi}\right]+\frac{1}{\lambda_{e}+2 \mu_{e}}\left[\boldsymbol{T}_{e, \boldsymbol{y}}[\hat{\boldsymbol{x}} \otimes \hat{\boldsymbol{x}}] \frac{e^{-i \kappa_{p}^{e} \hat{\boldsymbol{x}} \cdot \boldsymbol{y}}}{4 \pi}\right]\right) \boldsymbol{\psi}(\boldsymbol{y}) d s(\boldsymbol{y}) .
$$

The direct method is used to compute the farfield pattern of the solution to the forward problem while the indirect one is required to compute the Fréchet derivatives of the boundary to farfield operator. The direct method has the advantage to provide the boundary data which are needed to compute the boundary data of the Fréchet derivatives (see Remark 3.2). The connection between the two integral formulations given in Remark 2.2 is used to obtain the characterization of the adjoint operator (see the step 2 in the proof of Proposition 3.3)

\section{The Fréchet derivative and the adjoint operator}

From now on, we choose a fixed reference domain $\Omega_{\text {ref }}$ with a closed and orientable boundary $\Gamma_{\text {ref }}$ of class $\mathscr{C}^{2}$ at least and we consider variations generated by transformations of the form $\boldsymbol{x} \mapsto \boldsymbol{q}(\boldsymbol{x})$ of point $\boldsymbol{x}$ in the space $\mathbb{R}^{3}$, where $\boldsymbol{q}$ is a smooth vector function defined in a neighborhood of $\Gamma_{\text {ref }}$. We consider diffeomorphism $\boldsymbol{q}$ from $\Gamma_{\text {ref }}$ to $\Gamma_{\boldsymbol{q}}:=\left\{\boldsymbol{q}(\boldsymbol{x}) ; \boldsymbol{x} \in \Gamma_{\text {ref }}\right\}$, such that the surface $\Gamma_{\boldsymbol{q}}$ is still a smooth orientable boundary of a domain $\Omega_{\boldsymbol{q}}$ with same genus as $\Omega_{\text {ref }}$. We have the continuous embedding $H^{s}\left(\Gamma_{\text {ref }}, \mathbb{R}^{3}\right) \hookrightarrow \mathscr{C}^{1}\left(\Gamma_{\text {ref }}, \mathbb{R}^{3}\right)$ for any $s>2$ (see $[1$, pp. 98] and [47, pp. 50]). We choose $s>2$ and we define the following open set of admissible variations

$$
\mathcal{Q}:=\left\{\boldsymbol{q} \in H^{s}\left(\Gamma_{\text {ref }}, \mathbb{R}^{3}\right): \boldsymbol{q} \text { injective, } \operatorname{det}(D \boldsymbol{q}(\widehat{\mathbf{x}})) \neq 0 \text { for all } \widehat{\mathbf{x}} \in \Gamma_{\text {ref }}\right\} .
$$

By $\boldsymbol{n}_{\boldsymbol{q}}$ we denote the outward unit normal vector to $\Gamma_{\boldsymbol{q}}$ and, in what follows, we will distinguish the quantities related to the elastic transmission problem at the interface $\Gamma_{\boldsymbol{q}}$ through the index $\boldsymbol{q}$.

Let $F: \mathcal{Q} \rightarrow \boldsymbol{L}^{2}\left(\mathbb{S}^{2}\right)$ denote the operator which maps a parametrization $\boldsymbol{q} \in \mathcal{Q}$ of a boundary $\Gamma_{\boldsymbol{q}}$ to the far-field pattern $\boldsymbol{u}_{\boldsymbol{q}}^{\infty}$ corresponding to the incident field $\boldsymbol{u}^{\text {inc }}$. Using (2.5), the operator $F$ admits the factorization

$$
F(\boldsymbol{q})=\boldsymbol{u}_{\boldsymbol{q}}^{\infty}=\left(\begin{array}{ll}
\mathscr{F}_{\mathrm{N}}, \boldsymbol{q} & -\mathscr{F}_{\mathrm{D}, \boldsymbol{q}}
\end{array}\right)\left[\mathbf{I}_{o p, \boldsymbol{q}}^{\dagger}\right]^{-1}\left(\begin{array}{c}
\boldsymbol{u}_{\mid \Gamma_{q}}^{i n c} \\
\boldsymbol{T}_{e} \boldsymbol{u}_{\mid \Gamma_{q}}^{i n c}
\end{array}\right) .
$$

Theorem 3.1 (characterization of $F^{\prime}[\boldsymbol{q}]$ ) The mapping $F: \mathcal{Q} \rightarrow \boldsymbol{L}^{2}\left(\mathbb{S}^{2}\right)$ with $s>2$ is Fréchet differentiable at all $\boldsymbol{q} \in \mathcal{Q}$ for which $\Gamma_{\boldsymbol{q}}$ is of class $\mathscr{C}^{2}$, and the first derivative at $\boldsymbol{q}$ in the direction $\boldsymbol{\xi} \in H^{s}\left(\Gamma_{\mathrm{ref}}, \mathbb{R}^{3}\right)$ is given by

$$
F^{\prime}[\boldsymbol{q}] \boldsymbol{\xi}=\boldsymbol{v}_{\boldsymbol{q}, \boldsymbol{\xi}}^{\infty},
$$

where $\boldsymbol{v}_{\boldsymbol{q}, \boldsymbol{\xi}}^{\infty}$ is the far-field pattern of the solution $\left(\boldsymbol{v}_{\boldsymbol{q}, \boldsymbol{\xi}}^{i}, \boldsymbol{v}_{\boldsymbol{q}, \boldsymbol{\xi}}^{e}\right)$ to the Navier equations (2.1a)-(2.1b) in $\mathbb{R}^{3} \backslash \Gamma_{\boldsymbol{q}}$ that satisfies the Kupradze radiation condition and the transmission conditions on $\Gamma_{\boldsymbol{q}}$

$$
\left\{\begin{aligned}
\boldsymbol{v}_{\boldsymbol{q}, \boldsymbol{\xi}}^{i} & =\boldsymbol{v}_{\boldsymbol{q}, \boldsymbol{\xi}}^{e}+\boldsymbol{f}_{\boldsymbol{q}, \boldsymbol{\xi}}^{\prime} \\
\boldsymbol{T}_{i} \boldsymbol{v}_{\boldsymbol{q}, \boldsymbol{\xi}}^{i} & =\boldsymbol{T}_{e} \boldsymbol{v}_{\boldsymbol{q}, \boldsymbol{\xi}}^{e}+\boldsymbol{g}_{\boldsymbol{q}, \boldsymbol{\xi}}^{\prime}
\end{aligned}\right.
$$

with

$$
\begin{aligned}
\boldsymbol{f}_{\boldsymbol{q}, \boldsymbol{\xi}}^{\prime}= & -\left(\boldsymbol{\xi} \circ \boldsymbol{q}^{-1} \cdot \boldsymbol{n}_{\boldsymbol{q}}\right)\left(\frac{\partial}{\partial \boldsymbol{n}_{\boldsymbol{q}}} \boldsymbol{u}_{\boldsymbol{q}}^{i}-\frac{\partial}{\partial \boldsymbol{n}_{\boldsymbol{q}}}\left(\boldsymbol{u}_{\boldsymbol{q}}^{e}+\boldsymbol{u}^{i n c}\right)\right), \\
\boldsymbol{g}_{\boldsymbol{q}, \boldsymbol{\xi}}^{\prime}= & \left(\boldsymbol{\xi} \circ \boldsymbol{q}^{-1} \cdot \boldsymbol{n}_{\boldsymbol{q}}\right) \omega^{2}\left(\rho_{i} \boldsymbol{u}_{\boldsymbol{q}}^{i}-\rho_{e}\left(\boldsymbol{u}_{\boldsymbol{q}}^{e}+\boldsymbol{u}^{i n c}\right)\right) \\
& +\operatorname{div}_{\Gamma_{\boldsymbol{q}}}\left(\left(\boldsymbol{\xi} \circ \boldsymbol{q}^{-1} \cdot \boldsymbol{n}_{\boldsymbol{q}}\right) \mathrm{I}_{\boldsymbol{t}, \boldsymbol{q}}\left[\sigma^{i}\left(\boldsymbol{u}_{\boldsymbol{q}}^{i}\right)-\sigma^{e}\left(\boldsymbol{u}_{e}^{\boldsymbol{q}}+\boldsymbol{u}^{i n c}\right)\right] \mathrm{I}_{\boldsymbol{t}, \boldsymbol{q}}\right) .
\end{aligned}
$$


where for $a=i$, e we have set $\sigma^{a}(\boldsymbol{u})=\lambda_{a}(\operatorname{div} \boldsymbol{u}) \mathrm{I}_{3}+\mu_{a}\left([\boldsymbol{\nabla} \boldsymbol{u}]+{ }^{\mathrm{T}}[\boldsymbol{\nabla} \boldsymbol{u}]\right)$ and $\mathrm{I}_{\boldsymbol{t}, \boldsymbol{q}}=\mathrm{I}_{3}-\boldsymbol{n}_{\boldsymbol{q}} \otimes \boldsymbol{n}_{\boldsymbol{q}}$ and $\left(\boldsymbol{u}_{\boldsymbol{q}}^{i}, \boldsymbol{u}_{\boldsymbol{q}}^{e}\right)$ is the solution of the transmission problem (2.1a)-(2.1e) at the interface $\Gamma_{\boldsymbol{q}}$.

Proof. Let $\boldsymbol{q} \in \mathcal{Q}$ such that $\Gamma_{\boldsymbol{q}}$ is of class $\mathscr{C}^{2}$ at least. The solution $\left(\boldsymbol{u}_{\boldsymbol{q}}^{i}, \boldsymbol{u}_{\boldsymbol{q}}^{e}\right)$ of the transmission problem (2.1a)-(2.1e) at the interface $\Gamma_{\boldsymbol{q}}$ admits the following integral representation

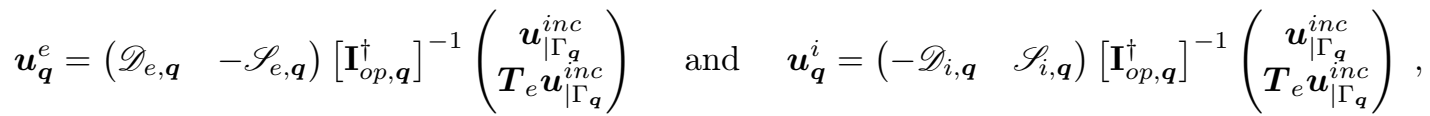

where $\mathscr{S}_{a, \boldsymbol{q}}$ and $\mathscr{D}_{a, \boldsymbol{q}}$, for $a=i, e$, are the single layer and double layer elastic potential operators [44, Eqs. (3.1) and (3.2), pp. 300-301]. The material derivatives of the boundary integral operators as well as those of the surface differential operators have been extensively analysed in [15] in the framework of Sobolev spaces. In [15, Section 4], it is shown that the material derivatives of the boundary integral operators with a pseudohomogeneous weakly or a strongly singular kernel exist at every order and the kernel of high order derivatives are still weakly or stongly singular. In [15, Section 5], this results are extended to boundary integral operators with a hypersingular kernel, as for example $N_{a}$ [15, Example 5.3], by expressing them in terms of surface differential operators and boundary integral operators with a weaklysingular kernel. The factorizations of the operators $D_{a}, D_{a}^{\prime}$ and $N_{a}$ can be found in [46, Lemmas 2.2 and 2.3]. Moreover the successive material derivatives at $\boldsymbol{q}$ in any direction $\boldsymbol{\xi}$ involves the first tangential derivatives of $\boldsymbol{\xi}$ only. By the chain and quotient rules, we conclude that the boundary to far-field operator $F$ is infinitely Fréchet differentiable.

Writing $\Omega_{\boldsymbol{q}}$ and $\Omega_{\boldsymbol{q}}^{c}$ as increasing unions of compact subsets $\Omega_{\boldsymbol{q}}=\bigcup_{i=1}^{\infty} K_{p}^{-}$and $\Omega_{\boldsymbol{q}}^{c}=\bigcup_{i=1}^{\infty} K_{p}^{+}$, we can establish the differentiability properties of the potential operators [15, Theorem 4.8] by analysing their restrictions to $K_{p}^{-} \cup K_{p}^{+}$for all $p \geq 1$. Let us fix $p \geq 1$. The kernels are $\mathscr{C}^{\infty}$-regular in $K_{p}^{-} \cup K_{p}^{+}$so that, by the chain and quotient rules, the potentials and the mapping $G: \boldsymbol{q} \in \mathcal{Q} \rightarrow\left(\boldsymbol{u}_{\boldsymbol{q}}^{i}, \boldsymbol{u}_{\boldsymbol{q}}^{e}\right) \in \boldsymbol{H}^{1}\left(K_{p}^{-}, \Delta_{i}^{*}\right) \times \boldsymbol{H}^{1}\left(K_{p}^{+}, \Delta_{e}^{*}\right)$ are infinitely Fréchet differentiable. Since we can interchange the differentiation with respect to $\boldsymbol{q}$ with the differentiation with respect to $\boldsymbol{x} \in K_{p}^{-} \cup K_{p}^{+}$, we deduce that the first Fréchet derivative $G^{\prime}[\boldsymbol{q}] \boldsymbol{\xi}=\left(\boldsymbol{v}_{\boldsymbol{q}, \boldsymbol{\xi}}^{i}, \boldsymbol{v}_{\boldsymbol{q}, \boldsymbol{\xi}}^{e}\right)$ for any $\boldsymbol{\xi} \in H^{s}\left(\Gamma_{\text {ref }}, \mathbb{R}^{3}\right) \subset \mathscr{C}^{1}\left(\Gamma_{\text {ref }}, \mathbb{R}^{3}\right)$ solves the Navier equations (2.1a)-(2.1b) in $K_{p}^{-} \cup K_{p}^{+}$. Collecting the results for all $p \geq 1$, we deduce $\left(\boldsymbol{v}_{\boldsymbol{q}, \boldsymbol{\xi}}^{i}, \boldsymbol{v}_{\boldsymbol{q}, \boldsymbol{\xi}}^{e}\right)$ solves the Navier equations (2.1a)-(2.1b) in $\Omega_{\boldsymbol{q}} \cup \Omega_{\boldsymbol{q}}^{c}$. However the first Fréchet derivatives, considered as functions in $\Omega_{\boldsymbol{q}} \cup \Omega_{\boldsymbol{q}}^{c}$, lose regularity. Since we can interchange the differentiation with respect to $\boldsymbol{q}$ and the passing to the limit $|\boldsymbol{x}| \rightarrow \infty$ we deduce that $\boldsymbol{v}_{\boldsymbol{q}, \boldsymbol{\xi}}^{e}$ satisfy the Kupradze radiations conditions $(2.1 \mathrm{e})$ and $\boldsymbol{v}_{\boldsymbol{q}, \boldsymbol{\xi}}^{\infty}$ is the farfield pattern of $\boldsymbol{v}_{\boldsymbol{q}, \boldsymbol{\xi}}^{e}$. The boundary data of $\boldsymbol{v}_{\boldsymbol{q}, \boldsymbol{\xi}}^{i}$ and $\boldsymbol{v}_{\boldsymbol{q}, \boldsymbol{\xi}}^{e}$ exists as distributions in $\boldsymbol{H}^{-\frac{1}{2}}\left(\Gamma_{\boldsymbol{q}}\right) \times \boldsymbol{H}^{-\frac{3}{2}}\left(\Gamma_{\boldsymbol{q}}\right)$. It remains to compute the transmission conditions satisfied by the first Fréchet derivative. We have

$$
\left\{\begin{array}{ll}
\boldsymbol{u}_{\boldsymbol{q}}^{i} \circ \boldsymbol{q}-\left(\boldsymbol{u}_{\boldsymbol{q}}^{e}+\boldsymbol{u}^{i n c}\right) \circ \boldsymbol{q} & \mathbf{0} \\
\left\{\sigma^{i}\left(\boldsymbol{u}_{\boldsymbol{q}}^{i}\right) \boldsymbol{n}_{\boldsymbol{q}}\right\} \circ \boldsymbol{q}-\left\{\sigma_{e}\left(\boldsymbol{u}_{\boldsymbol{q}}^{e}+\boldsymbol{u}^{i n c}\right) \boldsymbol{n}_{\boldsymbol{q}}\right\} \circ \boldsymbol{q}=\mathbf{0}
\end{array} \quad \text { on } \Gamma_{\mathrm{ref}} \text { and for all } \boldsymbol{q} \in \mathcal{Q}\right. \text {. }
$$

We will use the expansion of the gradient on the boundary $\Gamma_{\boldsymbol{q}}[47$, Eq. (2.5.208)]:

$$
\nabla \boldsymbol{u}=\nabla_{\Gamma_{\boldsymbol{q}}} \boldsymbol{u}_{\mid \Gamma_{\boldsymbol{q}}}+\boldsymbol{n}_{\boldsymbol{q}} \frac{\partial \boldsymbol{u}}{\boldsymbol{n}_{\boldsymbol{q}}}
$$

and the material derivative of the normal vector given by $[15$, Lemma 4.3$] \partial_{\boldsymbol{q}}\left\{\boldsymbol{n}_{\boldsymbol{q}} \circ \boldsymbol{q}\right\} \boldsymbol{\xi}=-\left(\left[\nabla_{\Gamma_{\boldsymbol{q}}}\left(\boldsymbol{\xi} \circ \boldsymbol{q}^{-1}\right)\right] \boldsymbol{n}_{\boldsymbol{q}}\right) \circ \boldsymbol{q}$.

- By differentiation with respect to $\boldsymbol{q}$, we have

$$
\begin{aligned}
\mathbf{0}=\partial_{\boldsymbol{q}}\left\{\boldsymbol{u}_{\boldsymbol{q}}^{i} \circ \boldsymbol{q}-\left(\boldsymbol{u}_{\boldsymbol{q}}^{e}+\boldsymbol{u}^{i n c}\right) \circ \boldsymbol{q}\right\} \boldsymbol{\xi}= & \boldsymbol{v}_{\boldsymbol{q}, \boldsymbol{\xi}}^{i} \circ \boldsymbol{q}+\boldsymbol{\xi} \cdot\left(\boldsymbol{\nabla} \boldsymbol{u}_{\boldsymbol{q}}^{i}\right)_{\Gamma_{\boldsymbol{q}}} \circ \boldsymbol{q}-\boldsymbol{v}_{\boldsymbol{q}, \boldsymbol{\xi}}^{e} \circ \boldsymbol{q}-\boldsymbol{\xi} \cdot\left(\boldsymbol{\nabla}\left(\boldsymbol{u}_{\boldsymbol{q}}^{e}+\boldsymbol{u}^{i n c}\right)\right)_{\Gamma_{\boldsymbol{q}}} \circ \boldsymbol{q} \\
= & \boldsymbol{v}_{\boldsymbol{q}, \boldsymbol{\xi}}^{i} \circ \boldsymbol{q}-\boldsymbol{v}_{\boldsymbol{q}, \boldsymbol{\xi}}^{e} \circ \boldsymbol{q} \\
& +\left(\boldsymbol{\xi} \cdot \boldsymbol{n}_{\boldsymbol{q}} \circ \boldsymbol{q}\right)\left(\frac{\partial}{\partial \boldsymbol{n}_{\boldsymbol{q}}} \boldsymbol{u}_{\boldsymbol{q}}^{i}-\frac{\partial}{\partial \boldsymbol{n}_{\boldsymbol{q}}}\left(\boldsymbol{u}_{\boldsymbol{q}}^{e}+\boldsymbol{u}^{i n c}\right)\right) \circ \boldsymbol{q} \\
& +\boldsymbol{\xi} \cdot\left\{\nabla_{\Gamma_{\boldsymbol{q}}} \boldsymbol{u}_{\boldsymbol{q}}^{i}-\nabla_{\Gamma_{\boldsymbol{q}}}\left(\boldsymbol{u}_{\boldsymbol{q}}^{e}+\boldsymbol{u}^{i n c}\right)\right\} \circ \boldsymbol{q}
\end{aligned}
$$


From the first transmission condition (2.1c) on $\Gamma_{\boldsymbol{q}}$ we deduce $\boldsymbol{\xi} \cdot\left[\nabla_{\Gamma_{\boldsymbol{q}}} \boldsymbol{u}_{\boldsymbol{q}}^{i}\right] \circ \boldsymbol{q}-\boldsymbol{\xi} \cdot\left[\nabla_{\Gamma_{\boldsymbol{q}}}\left(\boldsymbol{u}_{\boldsymbol{q}}^{e}+\boldsymbol{u}^{i n c}\right)\right] \circ \boldsymbol{q}=\mathbf{0}$, and the expression of $\boldsymbol{f}_{\boldsymbol{q}, \boldsymbol{\xi}}^{\prime}$.

- We also have

$$
\begin{aligned}
\mathbf{0}= & \partial_{\boldsymbol{q}}\left\{\left\{\sigma^{i}\left(\boldsymbol{u}_{\boldsymbol{q}}^{i}\right) \boldsymbol{n}_{\boldsymbol{q}}\right\} \circ \boldsymbol{q}-\left\{\sigma_{e}\left(\boldsymbol{u}_{\boldsymbol{q}}^{e}+\boldsymbol{u}^{i n c}\right) \boldsymbol{n}_{\boldsymbol{q}}\right\} \circ \boldsymbol{q}\right\} \boldsymbol{\xi} \\
= & \left\{\sigma^{i}\left(\boldsymbol{v}_{\boldsymbol{q}, \boldsymbol{\xi}}^{i}\right) \boldsymbol{n}_{\boldsymbol{q}}\right\} \circ \boldsymbol{q}-\left\{\sigma^{e}\left(\boldsymbol{v}_{\boldsymbol{q}, \boldsymbol{\xi}}^{e}\right) \boldsymbol{n}_{\boldsymbol{q}}\right\} \circ \boldsymbol{q} \\
& +\left\{\left[\left(\boldsymbol{\xi} \circ \boldsymbol{q}^{-1}\right) \cdot \boldsymbol{\nabla} \sigma^{i}\left(\boldsymbol{u}_{\boldsymbol{q}}^{i}\right)_{\Gamma_{\boldsymbol{q}}}\right] \boldsymbol{n}_{\boldsymbol{q}}-\left[\left(\boldsymbol{\xi} \circ \boldsymbol{q}^{-1}\right) \cdot \boldsymbol{\nabla} \sigma^{e}\left(\boldsymbol{u}_{\boldsymbol{q}}^{e}+\left.\boldsymbol{u}^{i n c}\right|_{\Gamma_{\boldsymbol{q}}}\right] \boldsymbol{n}_{\boldsymbol{q}}\right\} \circ \boldsymbol{q}\right. \\
& -\left\{\sigma^{i}\left(\boldsymbol{u}_{\boldsymbol{q}}^{i}\right)\left[\nabla_{\Gamma_{\boldsymbol{q}}}\left(\boldsymbol{\xi} \circ \boldsymbol{q}^{-1}\right)\right] \boldsymbol{n}_{\boldsymbol{q}}-\sigma_{e}\left(\boldsymbol{u}_{\boldsymbol{q}}^{e}+\boldsymbol{u}^{i n c}\right)\left[\nabla_{\Gamma_{\boldsymbol{q}}}\left(\boldsymbol{\xi} \circ \boldsymbol{q}^{-1}\right)\right] \boldsymbol{n}_{\boldsymbol{q}}\right\} \circ \boldsymbol{q},
\end{aligned}
$$

and

$$
\begin{aligned}
{\left[\left(\boldsymbol{\xi} \circ \boldsymbol{q}^{-1}\right) \cdot \nabla \sigma^{i}\left(\boldsymbol{u}_{\boldsymbol{q}}^{i}\right)_{\Gamma_{\boldsymbol{q}}}\right] \boldsymbol{n}_{\boldsymbol{q}}=} & \left(\boldsymbol{\xi} \circ \boldsymbol{q}^{-1} \cdot \boldsymbol{n}_{\boldsymbol{q}}\right)\left[\frac{\partial}{\partial \boldsymbol{n}_{\boldsymbol{q}}} \sigma^{i}\left(\boldsymbol{u}_{\boldsymbol{q}}^{i}\right)\right] \boldsymbol{n}_{\boldsymbol{q}}+\left[\left(\boldsymbol{\xi} \circ \boldsymbol{q}^{-1}\right) \cdot \nabla_{\Gamma_{\boldsymbol{q}}} \sigma^{i}\left(\boldsymbol{u}_{\boldsymbol{q}}^{i}\right)\right] \boldsymbol{n}_{q} \\
= & \left(\boldsymbol{\xi} \circ \boldsymbol{q}^{-1} \cdot \boldsymbol{n}_{\boldsymbol{q}}\right)\left[\frac{\partial}{\partial \boldsymbol{n}_{\boldsymbol{q}}} \sigma^{i}\left(\boldsymbol{u}_{\boldsymbol{q}}^{i}\right)\right] \boldsymbol{n}_{\boldsymbol{q}}+\left(\boldsymbol{\xi} \circ \boldsymbol{q}^{-1}\right) \cdot \nabla_{\Gamma_{\boldsymbol{q}}}\left(\sigma^{i}\left(\boldsymbol{u}_{\boldsymbol{q}}^{i}\right) \boldsymbol{n}_{q}\right) \\
& -\sigma^{i}\left(\boldsymbol{u}_{\boldsymbol{q}}^{i}\right)\left(\left(\boldsymbol{\xi} \circ \boldsymbol{q}^{-1}\right) \cdot \nabla_{\Gamma_{\boldsymbol{q}}} \boldsymbol{n}_{\boldsymbol{q}}\right) .
\end{aligned}
$$

We develop in the same way $\left[\left(\boldsymbol{\xi} \circ \boldsymbol{q}^{-1}\right) \cdot \boldsymbol{\nabla} \sigma^{e}\left(\boldsymbol{u}_{\boldsymbol{q}}^{e}+\boldsymbol{u}^{i n c}\right)_{\Gamma_{\boldsymbol{q}}}\right] \boldsymbol{n}_{\boldsymbol{q}}$. We use the second boundary condition to simplify $\left(\boldsymbol{\xi} \circ \boldsymbol{q}^{-1}\right) \cdot \nabla_{\Gamma_{\boldsymbol{q}}}\left(\sigma^{i}\left(\boldsymbol{u}_{\boldsymbol{q}}^{i}\right) \boldsymbol{n}_{q}\right)-\left(\boldsymbol{\xi} \circ \boldsymbol{q}^{-1}\right) \cdot \nabla_{\Gamma_{\boldsymbol{q}}}\left(\sigma^{e}\left(\boldsymbol{u}_{\boldsymbol{q}}^{e}+\boldsymbol{u}^{i n c}\right) \boldsymbol{n}_{q}\right)=\mathbf{0}$. We finaly get

$$
\begin{aligned}
\mathbf{0}= & \left\{\sigma^{i}\left(\boldsymbol{v}_{\boldsymbol{q}, \boldsymbol{\xi}}^{i}\right) \boldsymbol{n}_{\boldsymbol{q}}\right\} \circ \boldsymbol{q}-\left\{\sigma^{e}\left(\boldsymbol{v}_{\boldsymbol{q}, \boldsymbol{\xi}}^{e}\right) \boldsymbol{n}_{\boldsymbol{q}}\right\} \circ \boldsymbol{q} \\
& +\left\{\left(\boldsymbol{\xi} \circ \boldsymbol{q}^{-1} \cdot \boldsymbol{n}_{\boldsymbol{q}}\right)\left[\frac{\partial}{\partial \boldsymbol{n}_{\boldsymbol{q}}} \sigma^{i}\left(\boldsymbol{u}_{\boldsymbol{q}}^{i}\right)\right] \boldsymbol{n}_{\boldsymbol{q}}-\left(\boldsymbol{\xi} \circ \boldsymbol{q}^{-1} \cdot \boldsymbol{n}_{\boldsymbol{q}}\right)\left[\frac{\partial}{\partial \boldsymbol{n}_{\boldsymbol{q}}} \sigma^{i}\left(\boldsymbol{u}_{\boldsymbol{q}}^{e}+\boldsymbol{u}^{i n c}\right)\right] \boldsymbol{n}_{\boldsymbol{q}}\right\} \circ \boldsymbol{q} \\
& -\left\{\sigma^{i}\left(\boldsymbol{u}_{\boldsymbol{q}}^{i}\right) \nabla_{\Gamma_{\boldsymbol{q}}}\left(\boldsymbol{\xi} \circ \boldsymbol{q}^{-1} \cdot \boldsymbol{n}_{\boldsymbol{q}}\right)-\sigma_{e}\left(\boldsymbol{u}_{\boldsymbol{q}}^{e}+\boldsymbol{u}^{i n c}\right) \nabla_{\Gamma_{\boldsymbol{q}}}\left(\boldsymbol{\xi} \circ \boldsymbol{q}^{-1} \cdot \boldsymbol{n}_{\boldsymbol{q}}\right)\right\} \circ \boldsymbol{q} .
\end{aligned}
$$

Now we use the following decomposition of the divergence on $\Gamma_{\boldsymbol{q}}$ [47, Eq. (2.5.210)]

$$
\operatorname{div} \boldsymbol{u}=\operatorname{div}_{\Gamma_{\boldsymbol{q}}} \boldsymbol{u}+\boldsymbol{n}_{\boldsymbol{q}} \cdot \frac{\partial \boldsymbol{u}}{\partial \boldsymbol{n}_{\boldsymbol{q}}} .
$$

We obtain

$$
\left[\frac{\partial}{\partial \boldsymbol{n}_{\boldsymbol{q}}} \sigma^{i}\left(\boldsymbol{u}_{\boldsymbol{q}}^{i}\right)\right] \boldsymbol{n}_{\boldsymbol{q}}=\operatorname{div} \sigma^{i}\left(\boldsymbol{u}_{\boldsymbol{q}}^{i}\right)_{\left.\right|_{\Gamma_{\boldsymbol{q}}}}-\operatorname{div}_{\Gamma_{\boldsymbol{q}}} \sigma^{i}\left(\boldsymbol{u}_{\boldsymbol{q}}^{i}\right)_{\Gamma_{\boldsymbol{q}}}=-\rho_{i} \omega^{2} \boldsymbol{u}_{\left.\boldsymbol{q}\right|_{\Gamma_{\boldsymbol{q}}} ^{i}}-\operatorname{div}_{\Gamma_{\boldsymbol{q}}} \sigma^{i}\left(\boldsymbol{u}_{\boldsymbol{q}}^{i}\right)_{\left.\right|_{\Gamma_{\boldsymbol{q}}}}
$$

and

$$
\left(\boldsymbol{\xi} \circ \boldsymbol{q}^{-1} \cdot \boldsymbol{n}_{\boldsymbol{q}}\right) \operatorname{div}_{\Gamma_{\boldsymbol{q}}} \sigma^{i}\left(\boldsymbol{u}_{\boldsymbol{q}}^{i}\right)_{\Gamma_{\boldsymbol{q}}}+\sigma^{i}\left(\boldsymbol{u}_{\boldsymbol{q}}^{i}\right)_{\left.\right|_{\Gamma_{\boldsymbol{q}}}} \nabla_{\Gamma_{\boldsymbol{q}}}\left(\boldsymbol{\xi} \circ \boldsymbol{q}^{-1} \cdot \boldsymbol{n}_{\boldsymbol{q}}\right)=\operatorname{div}_{\Gamma_{\boldsymbol{q}}}\left(\left(\boldsymbol{\xi} \circ \boldsymbol{q}^{-1} \cdot \boldsymbol{n}_{\boldsymbol{q}}\right) \sigma^{i}\left(\boldsymbol{u}_{\boldsymbol{q}}^{i}\right)_{\Gamma_{\boldsymbol{q}}}\right) .
$$

We use again the second transmission condition to simplify $\sigma^{i}\left(\boldsymbol{u}_{\boldsymbol{q}}^{i}\right) \boldsymbol{n}_{\boldsymbol{q}} \otimes \boldsymbol{n}_{\boldsymbol{q}}-\sigma^{e}\left(\boldsymbol{u}_{\boldsymbol{q}}^{e}+\boldsymbol{u}^{i n c}\right) \boldsymbol{n}_{\boldsymbol{q}} \otimes \boldsymbol{n}_{\boldsymbol{q}}=\mathbf{0}$ on $\Gamma_{\boldsymbol{q}}$ and then, $\boldsymbol{n}_{\boldsymbol{q}} \otimes \boldsymbol{n}_{\boldsymbol{q}}\left(\sigma^{i}\left(\boldsymbol{u}_{\boldsymbol{q}}^{i}\right)-\sigma^{e}\left(\boldsymbol{u}_{\boldsymbol{q}}^{e}+\boldsymbol{u}^{i n c}\right)\right) \mathrm{I}_{\boldsymbol{t}, \boldsymbol{q}}=\mathbf{0}$. Collecting all the results, it yields the expression of $\boldsymbol{g}_{\boldsymbol{q}, \boldsymbol{\xi}}^{\prime}$.

We have $\left(\boldsymbol{f}_{\boldsymbol{q}, \boldsymbol{\xi}}^{\prime}, \boldsymbol{g}_{\boldsymbol{q}, \boldsymbol{\xi}}^{\prime}\right) \in \boldsymbol{H}^{-\frac{1}{2}}\left(\Gamma_{\boldsymbol{q}}\right) \times \boldsymbol{H}^{-\frac{3}{2}}\left(\Gamma_{\boldsymbol{q}}\right)$. For smooth boundaries $\Gamma_{\boldsymbol{q}}$, the boundary integral equation system can be solved on the space of weaker regularity $\boldsymbol{H}^{-\frac{1}{2}}\left(\Gamma_{\boldsymbol{q}}\right) \times \boldsymbol{H}^{-\frac{3}{2}}\left(\Gamma_{\boldsymbol{q}}\right)$. Since the far-field operators $\mathscr{F}_{\mathrm{D}, \boldsymbol{q}}$ and $\mathscr{F}_{\mathrm{N}}, \boldsymbol{q}$ are smoothing operators, we still have $\boldsymbol{v}_{\boldsymbol{q}, \boldsymbol{\xi}}^{\infty} \in \boldsymbol{L}^{2}\left(\mathbb{S}^{2}\right)$ by using this characterization. The characterization of higher order derivatives requires additional regularity for the boundary $\Gamma_{\boldsymbol{q}}$. 
Remark 3.2 For numerical convenience, it will be useful to express the boundary data ${ }^{\top}\left(\boldsymbol{f}_{\boldsymbol{q}, \boldsymbol{\xi}}^{\prime}, \boldsymbol{g}_{\boldsymbol{q}, \boldsymbol{\xi}}^{\prime}\right)$ in terms of the boundary data ${ }^{\top}\left(\boldsymbol{u}_{\boldsymbol{q}}^{i}, \boldsymbol{T}_{i} \boldsymbol{u}_{\boldsymbol{q}}^{i}\right)$, which is solution to the system (2.5), and their tangential derivatives. We use the following rewriting of the traction trace operator on $\Gamma_{\boldsymbol{q}}$

$$
\begin{aligned}
\boldsymbol{T}_{a} \boldsymbol{u}_{\boldsymbol{q}}^{a}=\sigma^{a}\left(\boldsymbol{u}_{\boldsymbol{q}}^{a}\right) \boldsymbol{n}_{\boldsymbol{q}} & =2 \mu_{a} \mathcal{M}_{\boldsymbol{q}} \boldsymbol{u}_{\boldsymbol{q}}^{a}+\left(\lambda_{a}+2 \mu_{a}\right)\left(\operatorname{div} \boldsymbol{u}_{\boldsymbol{q}}^{a}\right) \boldsymbol{n}_{\boldsymbol{q}}-\mu_{a} \boldsymbol{n}_{\boldsymbol{q}} \times \operatorname{curl} \boldsymbol{u}_{\boldsymbol{q}}^{a} \\
& =\mu_{a}\left(\frac{\partial \boldsymbol{u}_{\boldsymbol{q}}^{a}}{\partial \boldsymbol{n}_{\boldsymbol{q}}}+\mathcal{M}_{\boldsymbol{q}} \boldsymbol{u}_{\boldsymbol{q}}^{a}\right)+\left(\lambda_{a}+\mu_{a}\right)\left(\operatorname{div} \boldsymbol{u}_{\boldsymbol{q}}^{a}\right) \boldsymbol{n}_{\boldsymbol{q}} \\
& =\left(\lambda_{a}+2 \mu_{a}\right) \frac{\partial \boldsymbol{u}_{\boldsymbol{q}}^{a}}{\partial \boldsymbol{n}_{\boldsymbol{q}}}-\lambda_{a} \mathcal{M}_{\boldsymbol{q}} \boldsymbol{u}_{\boldsymbol{q}}^{a}+\left(\lambda_{a}+\mu_{a}\right) \boldsymbol{n}_{\boldsymbol{q}} \times \operatorname{curl} \boldsymbol{u}_{\boldsymbol{q}}^{a}
\end{aligned}
$$

where $\mathcal{M}_{\boldsymbol{q}}$ is the tangential Günter derivative on $\Gamma_{\boldsymbol{q}}$ defined by $\mathcal{M}_{\boldsymbol{q}}=\left(\left[\nabla_{\Gamma_{\boldsymbol{q}}} \cdot\right] \boldsymbol{n}_{\boldsymbol{q}}-\boldsymbol{n}_{\boldsymbol{q}} \operatorname{div}_{\Gamma_{\boldsymbol{q}}}\right)[44$, Equation (1.13) and Theorem 1.3, pages 282-284]. These formulas together with (3.3) lead to

$$
\begin{gathered}
\boldsymbol{n}_{\boldsymbol{q}} \times\left(\boldsymbol{T}_{a} \boldsymbol{u}_{\boldsymbol{q}}^{a} \times \boldsymbol{n}_{\boldsymbol{q}}\right)=\mu_{a} \boldsymbol{n}_{\boldsymbol{q}} \times\left(\frac{\partial}{\partial \boldsymbol{n}_{\boldsymbol{q}}} \boldsymbol{u}_{\boldsymbol{q}}^{a} \times \boldsymbol{n}_{\boldsymbol{q}}\right)+\mu_{a}\left[\nabla_{\Gamma_{\boldsymbol{q}}} \boldsymbol{u}_{\boldsymbol{q}}^{a}\right] \boldsymbol{n}_{\boldsymbol{q}}, \\
\boldsymbol{n}_{\boldsymbol{q}} \cdot \boldsymbol{T}_{a} \boldsymbol{u}_{\boldsymbol{q}}^{a}=\left(\lambda_{a}+2 \mu_{a}\right) \boldsymbol{n}_{\boldsymbol{q}} \cdot \frac{\partial \boldsymbol{u}_{\boldsymbol{q}}^{a}}{\partial \boldsymbol{n}_{\boldsymbol{q}}}+\lambda_{a} \operatorname{div}_{\Gamma_{\boldsymbol{q}}} \boldsymbol{u}_{\boldsymbol{q}}^{a},
\end{gathered}
$$

and

$$
\boldsymbol{n}_{\boldsymbol{q}} \cdot \boldsymbol{T}_{a} \boldsymbol{u}_{\boldsymbol{q}}^{a}=-2 \mu_{a} \operatorname{div}_{\Gamma_{\boldsymbol{q}}} \boldsymbol{u}_{\boldsymbol{q}}^{a}+\left(\lambda_{a}+2 \mu_{a}\right) \operatorname{div} \boldsymbol{u}_{\boldsymbol{q}}^{a} .
$$

We set $\boldsymbol{\xi}_{\boldsymbol{n}_{\boldsymbol{q}}}=\left(\boldsymbol{\xi} \circ \boldsymbol{q}^{-1} \cdot \boldsymbol{n}_{\boldsymbol{q}}\right)$ and $\mathrm{I}_{\boldsymbol{n}, \boldsymbol{q}}=\boldsymbol{n}_{\boldsymbol{q}} \otimes \boldsymbol{n}_{\boldsymbol{q}}$, then we have

$$
\left(\begin{array}{c}
\boldsymbol{f}_{\boldsymbol{q}, \boldsymbol{\xi}}^{\prime} \\
\boldsymbol{g}_{\boldsymbol{q}, \boldsymbol{\xi}}^{\prime}
\end{array}\right)=\mathcal{B}[\boldsymbol{q}] \boldsymbol{\xi}:=\left(\begin{array}{c}
\mathcal{B}_{1}[\boldsymbol{q}] \boldsymbol{\xi} \\
\mathcal{B}_{2}[\boldsymbol{q}] \boldsymbol{\xi}
\end{array}\right)
$$

where

$$
\mathcal{B}_{1}[\boldsymbol{q}] \boldsymbol{\xi}=\boldsymbol{\xi}_{\boldsymbol{n}_{\boldsymbol{q}}}\left\{\left(\frac{\lambda_{i}}{\lambda_{i}+2 \mu_{i}}-\frac{\lambda_{e}}{\lambda_{e}+2 \mu_{e}}\right) \boldsymbol{n}_{\boldsymbol{q}} \operatorname{div}_{\Gamma_{\boldsymbol{q}}} \boldsymbol{u}_{\boldsymbol{q}}^{i}+\left[\left(\frac{1}{\lambda_{e}+2 \mu_{e}}-\frac{1}{\lambda_{i}+2 \mu_{i}}\right) \mathrm{I}_{\boldsymbol{n}, \boldsymbol{q}} \boldsymbol{T}_{i} \boldsymbol{u}_{\boldsymbol{q}}^{i}+\left(\frac{1}{\mu_{e}}-\frac{1}{\mu_{i}}\right) \mathrm{I}_{\boldsymbol{t}, \boldsymbol{q}} \boldsymbol{T}_{i} \boldsymbol{u}_{\boldsymbol{q}}^{i}\right]\right\}
$$

and

$$
\mathcal{B}_{2}[\boldsymbol{q}] \boldsymbol{\xi}=\boldsymbol{\xi}_{\boldsymbol{n}_{\boldsymbol{q}}}\left(\rho_{i}-\rho_{e}\right) \omega^{2} \boldsymbol{u}_{\boldsymbol{q}}^{i}+\operatorname{div}_{\Gamma_{\boldsymbol{q}}}\left(\boldsymbol{\xi}_{\boldsymbol{n}_{\boldsymbol{q}}} \sigma_{\boldsymbol{t}}\left(\boldsymbol{u}_{\boldsymbol{q}}^{i}\right)\right)+\operatorname{div}_{\Gamma_{\boldsymbol{q}}}\left(\boldsymbol{\xi}_{\boldsymbol{n}_{\boldsymbol{q}}} \sigma_{\boldsymbol{n}}\left(T_{i} \boldsymbol{u}_{\boldsymbol{q}}^{i}\right)\right)
$$

with

$$
\sigma_{\boldsymbol{t}}\left(\boldsymbol{u}_{\boldsymbol{q}}^{i}\right)=\left(\frac{2 \lambda_{i} \mu_{i}}{\lambda_{i}+2 \mu_{i}}-\frac{2 \lambda_{e} \mu_{e}}{\lambda_{e}+2 \mu_{e}}\right)\left(\operatorname{div}_{\Gamma_{\boldsymbol{q}}} \boldsymbol{u}_{\boldsymbol{q}}^{i}\right) \mathrm{I}_{\boldsymbol{t}, \boldsymbol{q}}+\left(\mu_{i}-\mu_{e}\right) \mathrm{I}_{\boldsymbol{t}, \boldsymbol{q}}\left(\left[\nabla_{\Gamma_{\boldsymbol{q}}} \boldsymbol{u}_{\boldsymbol{q}}^{i}\right]+{ }^{\mathrm{T}}\left[\nabla_{\Gamma_{\boldsymbol{q}}} \boldsymbol{u}_{\boldsymbol{q}}^{i}\right]\right) \mathrm{I}_{\boldsymbol{t}, \boldsymbol{q}}
$$

and

$$
\sigma_{\boldsymbol{n}}\left(T_{i} \boldsymbol{u}_{\boldsymbol{q}}^{i}\right)=\left(\frac{\lambda_{i}}{\lambda_{i}+2 \mu_{i}}-\frac{\lambda_{e}}{\lambda_{e}+2 \mu_{e}}\right)\left(\boldsymbol{n}_{\boldsymbol{q}} \cdot T_{i} \boldsymbol{u}_{\boldsymbol{q}}^{i}\right) \mathrm{I}_{\boldsymbol{t}, \boldsymbol{q}} .
$$

An interesting feature of these formulas is that they make appear the contrasts between the interior and exterior values of the Lamé parameters. Numerical experiments are presented in Table 3 and attest the theoretical results.

With the objective to use Gauß-Newton iterations we need to compute the adjoint operator $F^{\prime}[\boldsymbol{q}]_{\mid \boldsymbol{L}^{2}}^{*}$ of $F^{\prime}[\boldsymbol{q}]$ for the complex $\boldsymbol{L}^{2}$ inner product (not to be mistaken the $\boldsymbol{L}^{2}$ duality product mentioned in Remark 2.2).

Proposition 3.3 (characterization of the adjoint $F^{\prime}[\boldsymbol{q}]_{\mid L^{2}}^{*}$ ) Let $\boldsymbol{q} \in \mathcal{Q}$ and

$$
\boldsymbol{u}_{\boldsymbol{h}}^{i n c}(\boldsymbol{y}):=\int_{\mathbb{S}^{2}}\left(\frac{e^{-i \kappa_{s}^{e} \hat{\boldsymbol{x}} \cdot \boldsymbol{y}}}{4 \pi \mu_{e}}(\widehat{\mathbf{x}} \times \boldsymbol{h}(\widehat{\mathbf{x}})) \times \widehat{\mathbf{x}}+\frac{e^{-i \kappa_{p}^{e} \hat{\boldsymbol{x}} \cdot \boldsymbol{y}}}{4 \pi\left(\lambda_{e}+2 \mu_{e}\right)}(\widehat{\mathbf{x}} \cdot \boldsymbol{h}(\widehat{\mathbf{x}})) \widehat{\mathbf{x}}\right) d s(\widehat{\mathbf{x}}), \quad \boldsymbol{y} \in \mathbb{R}^{3}
$$


denote the vector Herglotz function with density $\boldsymbol{h} \in \boldsymbol{L}^{2}\left(\mathbb{S}^{2}\right)$ and $\boldsymbol{u}_{\boldsymbol{q}, \overline{\boldsymbol{h}}}^{i}$ the interior wave solution to the elastodynamic transmission problem at the interface $\Gamma_{\boldsymbol{q}}$ with the incident wave $\boldsymbol{u}_{\overline{\boldsymbol{h}}}^{\text {inc }}$. Then the $\boldsymbol{L}^{2}$-adjoint of $F^{\prime}[\boldsymbol{q}]$ is defined by

$$
\begin{aligned}
F^{\prime}[\boldsymbol{q}]_{\mid \boldsymbol{L}^{2}}^{*} \boldsymbol{h}=J_{\boldsymbol{q}}\left(\boldsymbol{n}_{\boldsymbol{q}} \operatorname{Re}\{\right. & \left(\frac{1}{\lambda_{i}+2 \mu_{i}}-\frac{1}{\lambda_{e}+2 \mu_{e}}\right)\left(\boldsymbol{n}_{\boldsymbol{q}} \cdot \boldsymbol{T}_{i} \overline{\boldsymbol{u}_{\boldsymbol{q}}^{i}}\right)\left(\boldsymbol{n}_{\boldsymbol{q}} \cdot \boldsymbol{T}_{i} \overline{\boldsymbol{u}_{\boldsymbol{q}, \overline{\boldsymbol{h}}}^{i}}\right) \\
& +\left(\frac{\lambda_{e}}{\lambda_{e}+2 \mu_{e}}-\frac{\lambda_{i}}{\lambda_{i}+2 \mu_{i}}\right)\left(\operatorname{div}_{\Gamma_{\boldsymbol{q}}} \overline{\boldsymbol{u}_{\boldsymbol{q}}^{i}}\right)\left(\boldsymbol{n}_{\boldsymbol{q}} \cdot \boldsymbol{T}_{i} \overline{\boldsymbol{u}_{\boldsymbol{q}, \overline{\boldsymbol{h}}}^{i}}\right) \\
& +\left(\frac{1}{\mu_{i}}-\frac{1}{\mu_{e}}\right)\left(\boldsymbol{T}_{i} \overline{\boldsymbol{u}_{\boldsymbol{q}}^{i}} \times \boldsymbol{n}_{\boldsymbol{q}}\right) \cdot\left(\boldsymbol{T}_{i} \overline{\boldsymbol{u}_{\boldsymbol{q}, \overline{\boldsymbol{h}}}^{i}} \times \boldsymbol{n}_{\boldsymbol{q}}\right) \\
& +\left(\rho_{i}-\rho_{e}\right) \omega^{2} \overline{\boldsymbol{u}_{\boldsymbol{q}}^{i}} \cdot \overline{\boldsymbol{u}_{\boldsymbol{q}, \overline{\boldsymbol{h}}}^{i}}-\sigma_{\boldsymbol{t}}\left(\overline{\boldsymbol{u}_{\boldsymbol{q}}^{i}}\right): \nabla_{\Gamma_{\boldsymbol{q}}} \overline{\boldsymbol{u}_{\boldsymbol{q}, \overline{\boldsymbol{h}}}^{i}} \\
& \left.\left.+\left(\frac{\lambda_{e}}{\lambda_{e}+2 \mu_{e}}-\frac{\lambda_{i}}{\lambda_{i}+2 \mu_{i}}\right)\left(\boldsymbol{n}_{\boldsymbol{q}} \cdot \boldsymbol{T}_{i} \overline{\boldsymbol{u}_{\boldsymbol{q}}^{i}}\right)\left(\operatorname{div}_{\Gamma_{\boldsymbol{q}}} \overline{\boldsymbol{u}_{\boldsymbol{q}, \overline{\boldsymbol{h}}}^{i}}\right)\right\}\right) \circ \boldsymbol{q},
\end{aligned}
$$

where $J_{\boldsymbol{q}}$ is the determinant of the Jacobian matrix of the change of variable $\widehat{\mathbf{x}} \mapsto \boldsymbol{q}(\widehat{\mathbf{x}})$ and, for two $(3 \times 3)$ matrices $A$ and $B$ whose columns are denoted respectively by $\left(a_{1}, a_{2}, a_{3}\right)$ and $\left(b_{1}, b_{2}, b_{3}\right)$, we have set $A: B=$ $a_{1} \cdot b_{1}+a_{2} \cdot b_{2}+a_{3} \cdot b_{3}$.

Proof. The proof consists of three steps:

1. factorization of $F^{\prime}[\boldsymbol{q}]$ and $F^{\prime}[\boldsymbol{q}]_{\left.\right|_{L^{2}} ^{*}}^{*}$ : Due to (2.9), Theorem 3.1 and Remark $3.2 F^{\prime}[\boldsymbol{q}]$ has a factorization

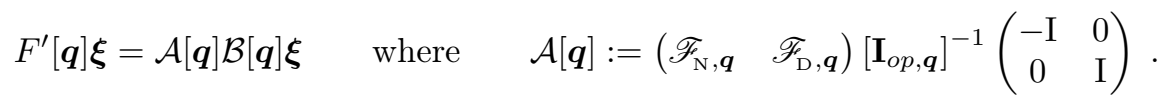

Let us denote by $(\mathcal{A}[\boldsymbol{q}])_{\left.\right|_{L^{2}}}^{*}$ and $(\mathcal{B}[\boldsymbol{q}])_{\mid L^{2}}^{*}$ the adjoints of $\mathcal{A}[\boldsymbol{q}]$ and $\mathcal{B}[\boldsymbol{q}]$ with respect to the $L^{2}$ inner products. Therefore, the adjoint of $F^{\prime}[\boldsymbol{q}]$ has the factorization

$$
F^{\prime}[\boldsymbol{q}]_{L^{2}}^{*} \boldsymbol{h}=(\mathcal{B}[\boldsymbol{q}])_{\boldsymbol{L}^{2}}^{*}(\mathcal{A}[\boldsymbol{q}])_{\mathbf{L}^{2}}^{*} \boldsymbol{h},
$$

and it remains to characterize $(\mathcal{A}[\boldsymbol{q}])_{\boldsymbol{L}^{2}}^{*}$ and $(\mathcal{B}[\boldsymbol{q}])_{\boldsymbol{L}^{2}}^{*}$

2. characterization of $\mathcal{A}[\boldsymbol{q}]_{\boldsymbol{L}^{2}}^{*}$ : Let us consider first the adjoint of $\mathscr{F}_{\mathrm{D}, \boldsymbol{q}}: \boldsymbol{H}^{t}\left(\Gamma_{\boldsymbol{q}}\right) \rightarrow \boldsymbol{L}^{2}\left(\mathbb{S}^{2}\right)$ for some $t \in \mathbb{R}$. For any $\boldsymbol{h} \in \boldsymbol{L}^{2}\left(\mathbb{S}^{2}\right)$ we have

$$
\left(\left.\mathscr{F}_{\mathrm{D}, \boldsymbol{q}}^{*}\right|_{L^{2}} \boldsymbol{h}\right)(\boldsymbol{y})=\frac{1}{4 \pi} \int_{\mathbb{S}^{2}}\left[\frac{e^{i \kappa_{\mathrm{e}} \widehat{\mathbf{x}} \cdot \boldsymbol{y}}}{\mu_{e}}\left[\mathrm{I}_{\mathbb{R}^{3}}-\hat{\boldsymbol{x}} \otimes \hat{\boldsymbol{x}}\right]+\frac{e^{i \kappa_{p}^{e} \hat{\boldsymbol{x}} \cdot \boldsymbol{y}}}{\lambda_{e}+2 \mu_{e}}[\hat{\boldsymbol{x}} \otimes \hat{\boldsymbol{x}}]\right] \boldsymbol{h}(\widehat{\mathbf{x}}) d s(\widehat{\mathbf{x}})=\overline{\boldsymbol{u}_{\hat{\boldsymbol{h}}}^{i n c}(\boldsymbol{y})} .
$$

The function $\overline{\boldsymbol{u}_{\bar{h}}^{i n c}(\boldsymbol{y})}$ is analytical on $\Gamma_{\boldsymbol{q}}$. We also obtain

$$
\left(\begin{array}{ll}
\mathscr{F}_{\mathrm{N}}, \boldsymbol{q} & \mathscr{F}_{\mathrm{D}}, \boldsymbol{q}
\end{array}\right)_{\mid L^{2}}^{*} \boldsymbol{h}=\left(\begin{array}{c}
\boldsymbol{T}_{e} \overline{\boldsymbol{u}_{\overline{\boldsymbol{h}}}^{\mathrm{inc}}} \\
\overline{\boldsymbol{u}_{\overline{\boldsymbol{h}}}^{\mathrm{inc}}}
\end{array}\right) .
$$

Therefore, using Remark 2.2 to pass from $\mathbf{I}_{o p}$ to $\mathbf{I}_{o p}^{\dagger}$, it follows that

$$
\begin{aligned}
(\mathcal{A}[\boldsymbol{q}])_{\mid \boldsymbol{L}^{2}}^{*} \boldsymbol{h} & =\left(\begin{array}{cc}
-\mathrm{I} & 0 \\
0 & \mathrm{I}
\end{array}\right) \overline{\left[\mathbf{I}_{o p, \boldsymbol{q}}\right]_{\mid \boldsymbol{L}^{2}}^{t-1}\left(\begin{array}{c}
\boldsymbol{T}_{e} \boldsymbol{u}_{\bar{h}}^{\text {inc }} \\
\boldsymbol{u}_{\bar{h}}^{\text {inc }}
\end{array}\right)} \\
& =\left(\begin{array}{cc}
0 & -\mathrm{I} \\
\mathrm{I} & 0
\end{array}\right) \overline{\left[\mathbf{I}_{o p, \boldsymbol{q}}^{\dagger}\right]^{-1}\left(\begin{array}{c}
\boldsymbol{u}_{\overline{\boldsymbol{h}}}^{\text {inc }} \\
\boldsymbol{T}_{e} \boldsymbol{u}_{\overline{\boldsymbol{h}}}^{\text {inc }}
\end{array}\right)}=\left(\begin{array}{c}
-\boldsymbol{T}_{i}^{\overline{\boldsymbol{u}_{\boldsymbol{q}, \bar{h}}^{i}}} \\
\overline{\boldsymbol{u}_{\boldsymbol{q}, \bar{h}}^{i}}
\end{array}\right)
\end{aligned}
$$


where we have used $(2.5)$ in the last line.

3. characterization of $\mathcal{B}[\boldsymbol{q}]_{\boldsymbol{L}^{2}}^{*}$ : We use

$$
\begin{aligned}
\left\langle\mathcal{B}[\boldsymbol{q}]_{\mid L^{2}}^{*}\left(\begin{array}{c}
-\boldsymbol{T}_{i} \overline{\boldsymbol{u}_{\boldsymbol{q}, \overline{\boldsymbol{h}}}^{i}} \\
\overline{\boldsymbol{u}_{\boldsymbol{q}, \overline{\boldsymbol{h}}}^{i}}
\end{array}\right), \boldsymbol{\xi}\right\rangle_{L^{2}\left(\Gamma_{\mathrm{ref}}, \mathbb{R}^{3}\right)} & =\operatorname{Re}\left\langle\left(\begin{array}{c}
-\boldsymbol{T}_{i} \overline{\boldsymbol{u}_{\boldsymbol{q}, \overline{\boldsymbol{h}}}^{i}} \\
\overline{\boldsymbol{u}_{\boldsymbol{q}, \overline{\boldsymbol{h}}}^{i}}
\end{array}\right), \mathcal{B}[\boldsymbol{q}] \boldsymbol{\xi}\right\rangle_{\boldsymbol{L}^{2}\left(\Gamma_{\boldsymbol{q}}\right) \times \boldsymbol{L}^{2}\left(\Gamma_{\boldsymbol{q}}\right)} \\
& =-\operatorname{Re}\left\langle\boldsymbol{T}_{i}^{\overline{\boldsymbol{u}_{\boldsymbol{q}, \bar{h}}^{i}}}, \mathcal{B}_{1}[\boldsymbol{q}] \boldsymbol{\xi}\right\rangle_{\boldsymbol{L}^{2}\left(\Gamma_{\boldsymbol{q}}\right)}+\operatorname{Re}\left\langle\overline{\boldsymbol{u}_{\boldsymbol{q}, \overline{\boldsymbol{h}}}^{i}}, \mathcal{B}_{2}[\boldsymbol{q}] \boldsymbol{\xi}\right\rangle_{\boldsymbol{L}^{2}\left(\Gamma_{\boldsymbol{q}}\right)} .
\end{aligned}
$$

For $\mathcal{B}_{1}[\boldsymbol{q}]$ we obtain

$$
\begin{aligned}
-\operatorname{Re}\left\langle\boldsymbol{T}_{i} \overline{\boldsymbol{u}_{\boldsymbol{q}, \overline{\boldsymbol{h}}}^{i}}, \mathcal{B}_{1}[\boldsymbol{q}] \boldsymbol{\xi}\right\rangle_{\boldsymbol{L}^{2}\left(\Gamma_{\boldsymbol{q}}\right)}=-\int_{\Gamma_{\boldsymbol{q}}}\left(\boldsymbol{\xi} \circ \boldsymbol{q}^{-1} \cdot \boldsymbol{n}_{\boldsymbol{q}}\right) \operatorname{Re}\{ & \left(\frac{1}{\lambda_{e}+2 \mu_{e}}-\frac{1}{\lambda_{i}+2 \mu_{i}}\right) \boldsymbol{T}_{i} \overline{\boldsymbol{u}_{\boldsymbol{q}, \overline{\boldsymbol{h}}}^{i}} \cdot \overline{\mathrm{I}_{\boldsymbol{n}, \boldsymbol{q}} \boldsymbol{T}_{i} \boldsymbol{u}_{\boldsymbol{q}}^{i}} \\
& +\left(\frac{1}{\mu_{e}}-\frac{1}{\mu_{i}}\right) \boldsymbol{T}_{i} \overline{\boldsymbol{u}_{\boldsymbol{q}, \overline{\boldsymbol{h}}}^{i}} \cdot \overline{\mathrm{I}_{\boldsymbol{t}, \boldsymbol{q}} \boldsymbol{T}_{i} \boldsymbol{u}_{\boldsymbol{q}}^{i}} \\
& \left.+\left(\frac{\lambda_{i}}{\lambda_{i}+2 \mu_{i}}-\frac{\lambda_{e}}{\lambda_{e}+2 \mu_{e}}\right) \boldsymbol{T}_{i} \overline{\boldsymbol{u}_{\boldsymbol{q}, \overline{\boldsymbol{h}}}^{i}} \cdot \overline{\boldsymbol{n}_{\boldsymbol{q}} \operatorname{div}_{\Gamma_{\boldsymbol{q}}} \boldsymbol{u}_{\boldsymbol{q}}^{i}}\right\} d s .
\end{aligned}
$$

We use a change of variable to pass from an integral over $\Gamma_{\boldsymbol{q}}$ to an integral over $\Gamma_{\text {ref }}$ and we finaly get

$$
\begin{aligned}
-\operatorname{Re}\left\langle\boldsymbol{T}_{i} \overline{\boldsymbol{u}_{\boldsymbol{q}, \overline{\boldsymbol{h}}}^{i}}, \mathcal{B}_{1}[\boldsymbol{q}] \boldsymbol{\xi}\right\rangle_{\boldsymbol{L}^{2}\left(\Gamma_{\boldsymbol{q}}\right)}=\int_{\Gamma_{\mathrm{ref}}} \boldsymbol{\xi} \cdot J_{\boldsymbol{q}}\left(\boldsymbol{n}_{\boldsymbol{q}} \operatorname{Re}\{\right. & \left(\frac{1}{\lambda_{i}+2 \mu_{i}}-\frac{1}{\lambda_{e}+2 \mu_{e}}\right)\left(\boldsymbol{n}_{\boldsymbol{q}} \cdot \boldsymbol{T}_{i} \overline{\boldsymbol{u}_{\boldsymbol{q}, \overline{\boldsymbol{h}}}^{i}}\right)\left(\boldsymbol{n}_{\boldsymbol{q}} \cdot \boldsymbol{T}_{i} \overline{\boldsymbol{u}_{\boldsymbol{q}}^{i}}\right) \\
& +\left(\frac{1}{\mu_{i}}-\frac{1}{\mu_{e}}\right)\left(\boldsymbol{T}_{i} \overline{\boldsymbol{u}_{\boldsymbol{q}, \overline{\boldsymbol{h}}}^{i}} \times \boldsymbol{n}_{\boldsymbol{q}}\right) \cdot\left(\boldsymbol{T}_{i} \overline{\boldsymbol{u}_{\boldsymbol{q}}^{i}} \times \boldsymbol{n}_{\boldsymbol{q}}\right) \\
& \left.\left.+\left(\frac{\lambda_{e}}{\lambda_{e}+2 \mu_{e}}-\frac{\lambda_{i}}{\lambda_{i}+2 \mu_{i}}\right)\left(\boldsymbol{n}_{\boldsymbol{q}} \cdot \boldsymbol{T}_{i} \overline{\boldsymbol{u}_{\boldsymbol{q}, \overline{\boldsymbol{h}}}^{i}}\right)\left(\operatorname{div}_{\Gamma_{\boldsymbol{q}}} \overline{\boldsymbol{u}_{\boldsymbol{q}}^{i}}\right)\right\}\right) \circ \boldsymbol{q} d s .
\end{aligned}
$$

For $\mathcal{B}_{2}[\boldsymbol{q}]$ we obtain

$$
\begin{aligned}
\operatorname{Re}\left\langle\overline{\boldsymbol{u}_{\boldsymbol{q}, \overline{\boldsymbol{h}}}^{i}}, \mathcal{B}_{2}[\boldsymbol{q}] \boldsymbol{\xi}\right\rangle_{\boldsymbol{L}^{2}\left(\Gamma_{\boldsymbol{q}}\right)}= & \int_{\Gamma_{\boldsymbol{q}}}\left(\boldsymbol{\xi} \circ \boldsymbol{q}^{-1} \cdot \boldsymbol{n}_{\boldsymbol{q}}\right) \operatorname{Re}\left\{\left(\rho_{i}-\rho_{e}\right) \omega^{2} \overline{\boldsymbol{u}_{\boldsymbol{q}, \overline{\boldsymbol{h}}}^{i}} \cdot \overline{\boldsymbol{u}_{\boldsymbol{q}}^{i}}\right\} d s \\
& +\int_{\Gamma_{\boldsymbol{q}}} \operatorname{Re}\left\{\overline{\boldsymbol{u}_{\boldsymbol{q}, \overline{\boldsymbol{h}}}^{i}} \cdot \operatorname{div}_{\Gamma_{\boldsymbol{q}}}\left(\left(\boldsymbol{\xi} \circ \boldsymbol{q}^{-1} \cdot \boldsymbol{n}_{\boldsymbol{q}}\right) \sigma_{\boldsymbol{t}}\left(\overline{\boldsymbol{u}_{\boldsymbol{q}}^{i}}\right)\right)\right. \\
& \left.\quad+\overline{\boldsymbol{u}_{\boldsymbol{q}, \overline{\boldsymbol{h}}}^{i}} \cdot \operatorname{div}_{\Gamma_{\boldsymbol{q}}}\left(\left(\boldsymbol{\xi} \circ \boldsymbol{q}^{-1} \cdot \boldsymbol{n}_{\boldsymbol{q}}\right) \sigma_{\boldsymbol{n}}\left(T_{i} \overline{\boldsymbol{u}_{\boldsymbol{q}}^{i}}\right)\right)\right\} d s .
\end{aligned}
$$

We note that the vector columns and the vector lines $\sigma_{\boldsymbol{n}}\left(T_{i} \overline{\boldsymbol{u}_{\boldsymbol{q}}^{i}}\right)$ and $\sigma_{\boldsymbol{t}}\left(\overline{\boldsymbol{u}_{\boldsymbol{q}}^{i}}\right)$ are tangential fields and we can use the integration by part formula $\int_{\Gamma_{q}} \varphi_{1} \operatorname{div}_{\Gamma_{q}} \varphi_{2} d s=-\int_{\Gamma_{q}} \nabla_{\Gamma_{q}} \varphi_{1} \cdot \varphi_{2} d s$ for any scalar function $\varphi_{1}$ and any tangential vector function $\varphi_{2}$. We finaly get

$$
\begin{aligned}
& \operatorname{Re}\left\langle\overline{\boldsymbol{u}_{\boldsymbol{q}, \overline{\boldsymbol{h}}}^{i}}, \mathcal{B}_{2}[\boldsymbol{q}] \boldsymbol{\xi}\right\rangle_{\boldsymbol{L}^{2}\left(\Gamma_{\boldsymbol{q}}\right)}=\int_{\Gamma_{\mathrm{ref}}} \boldsymbol{\xi} \cdot J_{\boldsymbol{q}}\left(\boldsymbol{n}_{\boldsymbol{q}} \operatorname{Re}\left\{\left(\rho_{i}-\rho_{e}\right) \omega^{2} \overline{\boldsymbol{u}_{\boldsymbol{q}, \overline{\boldsymbol{h}}}^{i}} \cdot \overline{\boldsymbol{u}_{\boldsymbol{q}}^{i}}\right\}\right) \circ \boldsymbol{q} d s \\
& -\int_{\Gamma_{q}} \boldsymbol{\xi} \cdot J_{\boldsymbol{q}}\left(\boldsymbol { n } _ { \boldsymbol { q } } \operatorname { R e } \left\{\nabla_{\Gamma_{\boldsymbol{q}}} \overline{\boldsymbol{u}_{\boldsymbol{q}, \bar{h}}^{i}}: \sigma_{\boldsymbol{t}}\left(\overline{\boldsymbol{u}_{\boldsymbol{q}}^{i}}\right)\right.\right. \\
& \left.\left.+\nabla_{\Gamma_{\boldsymbol{q}}} \overline{\boldsymbol{u}_{\boldsymbol{q}, \overline{\boldsymbol{h}}}^{i}}: \sigma_{\boldsymbol{n}}\left(T_{i} \overline{\boldsymbol{u}_{\boldsymbol{q}}^{i}}\right)\right\}\right) \circ \boldsymbol{q} d s,
\end{aligned}
$$

with $\nabla_{\Gamma_{\boldsymbol{q}}} \overline{\boldsymbol{u}_{\boldsymbol{q}, \overline{\boldsymbol{h}}}^{i}}: \sigma_{\boldsymbol{n}}\left(T_{i} \overline{\boldsymbol{u}_{\boldsymbol{q}}^{i}}\right)=\left(\frac{\lambda_{i}}{\lambda_{i}+2 \mu_{i}}-\frac{\lambda_{e}}{\lambda_{e}+2 \mu_{e}}\right)\left(\boldsymbol{n}_{\boldsymbol{q}} \cdot T_{i} \overline{\boldsymbol{u}_{\boldsymbol{q}}^{i}}\right)\left(\nabla_{\Gamma_{\boldsymbol{q}}} \overline{\boldsymbol{u}_{\boldsymbol{q}, \overline{\boldsymbol{h}}}^{i}}: \mathrm{I}_{\boldsymbol{t}, \boldsymbol{q}}\right)$ and $\left(\nabla_{\Gamma_{\boldsymbol{q}}} \overline{\boldsymbol{u}_{\boldsymbol{q}, \overline{\boldsymbol{h}}}^{i}}: \mathrm{I}_{\boldsymbol{t}, \boldsymbol{q}}\right)=\operatorname{div}_{\Gamma_{\boldsymbol{q}}} \overline{\boldsymbol{u}_{\boldsymbol{q}, \overline{\boldsymbol{h}}}^{i}}$. 


\section{The inverse scattering algorithm}

In this section we present the inverse problem of interest and describe an inverse scattering algorithm when the unknown scatterer is diffeomorphic to a ball which means $\Gamma_{\text {ref }}=\mathbb{S}^{2}$.

We consider the scattering of $m \in \mathbb{N}^{*}$ incident plane waves of the form [3, Section 3$]$ or [22, section 3$]$ :

$$
\boldsymbol{u}_{\ell}^{i n c}(\boldsymbol{x})=\frac{1}{\mu_{e}} e^{i \kappa_{s}^{e} \boldsymbol{x} \cdot \boldsymbol{d}_{\ell}}\left(\boldsymbol{d}_{\ell} \times \boldsymbol{p}_{\ell}\right) \times \boldsymbol{d}_{\ell}+\frac{1}{\lambda_{e}+2 \mu_{e}} e^{i \kappa_{p}^{e} \boldsymbol{x} \cdot \boldsymbol{d}_{\ell}}\left(\boldsymbol{d}_{\ell} \cdot \boldsymbol{p}_{\ell}\right) \boldsymbol{d}_{\ell}
$$

where for $\ell=1, \ldots, m$ we have $\boldsymbol{d}_{\ell}, \boldsymbol{p}_{\ell} \in \mathbb{S}^{2}$. When the polarization $\boldsymbol{p}_{\ell} \cdot \boldsymbol{d}_{\ell}=0$, the incident plane wave $\boldsymbol{u}_{\ell}^{i n c}$ oscillate in a direction orthogonal to the direction of propagation. They are called shear waves. When $\boldsymbol{p}_{\ell}=\boldsymbol{d}_{\ell}$, the incident plane waves oscillate along the direction of propagation. Let $F_{\ell}: \mathcal{Q} \rightarrow \boldsymbol{L}^{2}\left(\mathbb{S}^{2}\right), \ell=1, \ldots, m$ denote the operator which maps a parametrization $\boldsymbol{q} \in \mathcal{Q}$ of a boundary $\Gamma_{\boldsymbol{q}}$ to the far-field pattern $\boldsymbol{u}_{\ell}^{\infty}$ corresponding to the incident field $\boldsymbol{u}_{\ell}^{i n c}$. These operators may be combined into one operator $\boldsymbol{F}: \mathcal{Q} \rightarrow \boldsymbol{L}^{2}\left(\mathbb{S}^{2}\right)^{m}$, $\boldsymbol{F}(\boldsymbol{q}):=\left(F_{1}(\boldsymbol{q}), \ldots, F_{m}(\boldsymbol{q})\right)^{\top}$. The inverse problem is formulated as follows: Given noisy far field measurements $\boldsymbol{u}_{1, \delta}^{\infty}, \ldots, \boldsymbol{u}_{m, \delta}^{\infty}$ solve for $\ell=1, \ldots, m$

$$
F_{\ell}\left(\boldsymbol{q}^{\delta}\right)=\boldsymbol{u}_{\ell, \delta}^{\infty}
$$

We also combine the measured far-field patterns into a vector $\boldsymbol{u}_{\delta}^{\infty}:=\left(\boldsymbol{u}_{1, \delta}^{\infty}, \ldots, \boldsymbol{u}_{m, \delta}^{\infty}\right)^{\top} \in \boldsymbol{L}^{2}\left(\mathbb{S}^{2}\right)^{m}$ such that the inverse problem can be written as

$$
\boldsymbol{F}\left(\boldsymbol{q}^{\delta}\right)=\boldsymbol{u}_{\delta}^{\infty}
$$

Here, $\delta$ denotes the noise level which is measured in the $\boldsymbol{L}^{2}\left(\mathbb{S}^{2}\right)$-norm, i.e. $\left\|\boldsymbol{u}_{\delta}^{\infty}-\boldsymbol{u}_{0}^{\infty}\right\|_{\boldsymbol{L}^{2}\left(\mathbb{S}^{2}\right)^{m}}<\delta$. To compute an approximate solution to (4.1) we use the IRGN method [5, 28, 29]. At each iteration step, the iterates of the IRGN method can be computed by

$$
\boldsymbol{q}_{N+1}^{\delta}:=\operatorname{argmin}_{\boldsymbol{q} \in \boldsymbol{H}^{s}}\left[\left\|\boldsymbol{F}\left(\boldsymbol{q}_{N}^{\delta}\right)+\boldsymbol{F}^{\prime}\left[\boldsymbol{q}_{N}^{\delta}\right]\left(\boldsymbol{q}-\boldsymbol{q}_{N}^{\delta}\right)-\boldsymbol{u}_{\delta}^{\infty}\right\|_{\boldsymbol{L}^{2}\left(\mathbb{S}^{2}\right)^{m}}^{2}+\alpha_{N}\left\|\boldsymbol{q}-\boldsymbol{q}_{0}\right\|_{\boldsymbol{H}^{s}\left(\mathbb{S}^{2}, \mathbb{R}^{3}\right)}^{2}\right] .
$$

Here $\boldsymbol{q}_{0}=\boldsymbol{q}_{0}^{\delta}$ is some initial guess, and the regularization parameters are chosen of the form $\alpha_{N}=\alpha_{0} \gamma^{-N}$ with $\gamma>1$, which provides logarithmic convergence rates of the IRGN method [28, 29, Theorem 4.9] when the stoping rule is given by the Morozov's discrepancy principle (5.1).

The updates $(\partial \boldsymbol{q})_{N}:=\boldsymbol{q}_{N+1}^{\delta}-\boldsymbol{q}_{N}^{\delta}$ are the unique solutions to the linear equations [5]

$$
\left(\alpha_{N} \mathrm{I}+\boldsymbol{F}^{\prime}\left[\boldsymbol{q}_{N}^{\delta}\right]_{\left.\right|_{L^{2}}}^{*} \boldsymbol{F}_{k}^{\prime}\left[\boldsymbol{q}_{N}^{\delta}\right]\right)(\partial \boldsymbol{q})_{N}^{\delta}=\sum_{k=1}^{m} \boldsymbol{F}_{k}^{\prime}\left[\boldsymbol{q}_{N}^{\delta}\right]_{\mid L^{2}}^{*}\left(\boldsymbol{u}_{k, \delta}^{\infty}-\boldsymbol{F}_{k}\left(\boldsymbol{q}_{N}^{\delta}\right)\right)+\alpha_{N}\left(\boldsymbol{q}_{0}^{\delta}-\boldsymbol{q}_{N}^{\delta}\right),
$$

which is solved by the conjugate gradient (CG) method.

In the special case where we seek to restore a star-shaped scattering object with respect to the origin, we can consider special parametrizations of the form

$$
\boldsymbol{q}=\mathcal{R} r \quad \text { with } \quad(\mathcal{R} r)(\widehat{\mathbf{x}}):=r(\widehat{\mathbf{x}}) \widehat{\mathbf{x}}, \quad \widehat{\mathbf{x}} \in \mathbb{S}^{2}
$$

with a function $r: \mathbb{S}^{2} \rightarrow(0, \infty)$. Then the function $r$ is uniquely determined by $\Gamma_{\boldsymbol{q}}$. In this case we set, for some $s>2, \mathcal{Q}_{\text {star }}:=\left\{r \in H^{s}(\Gamma, \mathbb{R}): r>0\right\}$. As $\mathcal{R}\left(\mathcal{Q}_{\text {star }}\right) \subset \mathcal{Q}$, we can define $F_{\text {star }}: \mathcal{Q}_{\text {star }} \rightarrow \boldsymbol{L}^{2}\left(\mathbb{S}^{2}\right)^{m}$ by

$$
F_{\text {star }}:=\boldsymbol{F} \circ \mathcal{R} \text {. }
$$

Then $F_{\text {star }}$ is injective if a star-shaped interface $\Gamma_{\boldsymbol{q}}$ is uniquely determined by the far field data $\boldsymbol{u}_{1}^{\infty}, \ldots, \boldsymbol{u}_{m}^{\infty}$. We obtain $F_{\text {star }}^{\prime}[r]_{\boldsymbol{L}^{2}}^{*} \boldsymbol{h}=\widehat{\mathrm{x}} \cdot \operatorname{Re}\{\ldots\} \circ \boldsymbol{q}$ where the expression in the curly brackets coincides with the one in Proposition 3.3. 
Fully discrete algorithm. To solve the forward problem and compute the Fréchet derivatives we use the fast spectral method proposed by the author in [46]. For any $n \in \mathbb{N}^{*}$, let $\mathbb{H}_{n}^{\mathbb{R}}$ be the $(n+1)^{2}$ finite dimensional space spanned by the orthonormal scalar real spherical harmonics of degree at most $n$ and let $\mathbb{Q}_{n}$ be the $3(n+1)^{2}-2$ finite dimensional space spanned by the orthonormal vector spherical harmonics of degree at most $n$. Let $\mathcal{O}_{n}$ and $\mathscr{L}_{n}$ be the projection operators on $\mathbb{Q}_{n}$ and $\mathbb{H}_{n}^{\mathbb{R}}$ defined in [20, Eq. (3.10)] and [19, Eq. (2.44)] respectively. In the sequel, we consider that the solution to the boundary integral equations systems and the unknown parametrization are approached by vector or scalar spherical harmonics of degree at most $n_{\text {sol }}$ and $n_{\text {star }}$, respectively. The far-field data are evaluated at $2\left(n_{\text {meas }}+1\right)^{2}$ quadrature points $\widehat{\mathbf{x}}={ }^{\mathrm{T}}\left(\cos \phi_{r} \sin \theta_{s}, \sin \phi_{r} \sin \theta_{s}, \cos \theta_{s}\right)$ with $\theta_{s}=\cos ^{-1} z_{s}$, where $z_{s}, s=1, \ldots, n_{m e a s}+1$, are the zeros of the Legendre polynomial of degree $n_{\text {meas }}+1$, and $\phi_{r}=\frac{r \pi}{n_{\text {meas }}+1}, r=0, \ldots, 2 n_{\text {meas }}+1$.

Following details in [46], we denote by $\left[\mathbf{I}_{o p}\right]_{n_{s o l}}$ the spectral approximation of the operator $\mathbf{I}_{o p}$ defined on the space $\mathbb{Q}_{n_{s o l}}^{2}$ and by $\left[\mathbf{I}_{o p}^{\dagger}\right]_{n_{s o l}}$ the spectral approximation of the operator $\mathbf{I}_{o p}^{\dagger}$. The discrete numerical scheme for solving the forward problem using the system (2.5) is: find $\boldsymbol{u}_{n_{s o l}}^{i}=\mathcal{O}_{n_{\text {sol }}} \boldsymbol{u}_{\boldsymbol{q}}^{i} \circ \boldsymbol{q} \in \mathbb{Q}_{n_{\text {sol }}}$ and $\boldsymbol{t}_{n_{\text {sol }}}^{i}=\mathcal{O}_{n_{\text {sol }}}\left(\boldsymbol{T}_{i} \boldsymbol{u}_{\boldsymbol{q}}^{i}\right) \circ \boldsymbol{q} \in \mathbb{Q}_{n_{\text {sol }}}$ such that

$$
\left[\mathbf{I}_{o p}^{\dagger}\right]_{n_{s o l}}\left(\begin{array}{l}
\boldsymbol{u}_{n_{s o l}}^{i} \\
\boldsymbol{t}_{n_{s o l}}^{i}
\end{array}\right)=\left(\begin{array}{c}
\mathcal{O}_{n_{s o l}} J_{\boldsymbol{q}} \boldsymbol{u}^{i n c} \circ \boldsymbol{q} \\
\mathcal{O}_{n_{s o l}} J_{\boldsymbol{q}}\left(\boldsymbol{T}_{e} \boldsymbol{u}^{i n c}\right) \circ \boldsymbol{q}
\end{array}\right)
$$

Setting $\left(\mathscr{F}_{\mathrm{N}, \boldsymbol{q}}-\mathscr{F}_{\mathrm{D}, \boldsymbol{q}}\right)_{n_{\text {meas }}}$ the spectral approximation of the elastic far-field operators defined from $\mathbb{Q}_{n_{s o l}}^{2}$ to $\mathbb{Q}_{n_{\text {meas }}}$, then the approximate farfield data is

$$
F_{\text {star }}(r)=\left(\begin{array}{ll}
\mathscr{F}_{\mathrm{N}}, \boldsymbol{q} & -\mathscr{F}_{\mathrm{D}}, \boldsymbol{q}
\end{array}\right)_{n_{\text {meas }}}\left(\begin{array}{l}
\boldsymbol{u}_{n_{\text {sol }}}^{i} \\
\boldsymbol{t}_{n_{\text {sol }}}^{i}
\end{array}\right) .
$$

The discrete numerical scheme for computing the Fréchet derivative of $F_{\text {star }}$ at $r$ in the direction $\nu$ using the system (2.9) is: find $\boldsymbol{\psi}_{n_{\text {sol }}}=\mathcal{O}_{n_{\text {sol }}} \boldsymbol{\psi}_{\boldsymbol{q}} \circ \boldsymbol{q} \in \mathbb{Q}_{n_{\text {sol }}}$ and $\boldsymbol{\varphi}_{n_{\text {sol }}}=\mathcal{O}_{n_{\text {sol }}} \boldsymbol{\varphi}_{\boldsymbol{q}} \circ \boldsymbol{q} \in \mathbb{Q}_{n_{\text {sol }}}$ such that

$$
\left[\mathbf{I}_{o p}\right]_{n_{s o l}}\left(\begin{array}{l}
\boldsymbol{\psi}_{n_{s o l}} \\
\boldsymbol{\varphi}_{n_{s o l}}
\end{array}\right)=\left(\begin{array}{c}
-\mathcal{O}_{n_{s o l}} J_{\boldsymbol{q}} \boldsymbol{f}_{\boldsymbol{q}, \boldsymbol{\xi}}^{\prime} \circ \boldsymbol{q} \\
\mathcal{O}_{n_{s o l}} J_{\boldsymbol{q}} \boldsymbol{g}_{\boldsymbol{q}, \boldsymbol{\xi}}^{\prime}{ }^{\circ} \boldsymbol{q}
\end{array}\right) \quad \text { where } \boldsymbol{\xi}=\mathcal{R} \nu
$$

Setting $\left(\mathscr{F}_{\mathrm{N}, \boldsymbol{q}} \quad \mathscr{F}_{\mathrm{D}, \boldsymbol{q}}\right)_{n_{\text {meas }}}$ the spectral approximation of the elastic far-field operators defined from $\mathbb{Q}_{n_{s o l}}^{2}$ to $\mathbb{Q}_{n_{\text {meas }}}$, then the approximate Fréchet derivative of the farfield data is

$$
F_{\text {star }}^{\prime}[r] \nu=\left(\begin{array}{ll}
\mathscr{F}_{\mathrm{N}}, \boldsymbol{q} & \mathscr{F}_{\mathrm{D}}, \boldsymbol{q}
\end{array}\right)_{n_{\text {meas }}}\left(\begin{array}{l}
\boldsymbol{\psi}_{n_{\text {sol }}} \\
\boldsymbol{\varphi}_{n_{\text {sol }}}
\end{array}\right) .
$$

The discrete solution space for the inverse problem (4.1) is $\mathbb{H}_{n_{s t a r}}^{\mathbb{R}}$ undowed with the norm of $H^{s}$ given in the appendix. We denote by $j_{L^{2} \rightarrow H^{s}}^{\mathbb{R}}$ the restriction of the isomorphism defined by (A.1) to real-valued functions and by $B_{n_{\text {star }}}^{-s}=\mathscr{L}_{n_{\text {star }}} j_{L^{2} \rightarrow H^{s}}^{\mathbb{R}}$ its discrete approximation. In practice, we represent $\nu$ by the vector $B_{n_{s t a r}}^{-s}$ and we solve (4.2) in $L^{2}[29$, Chapter 5]. The complete algorithm is the following.

1. Choose an initial guess $\boldsymbol{q}_{0}^{\delta}=\boldsymbol{q}_{0}=\mathcal{R} r_{0}$.

2. For the parametrization $\boldsymbol{q}_{N}^{\delta}=\mathcal{R} r_{N}^{\delta}: \Gamma_{\text {ref }} \rightarrow \mathbb{R}^{3}$ of the current reconstruction $\Gamma_{N}^{\delta}:=\boldsymbol{q}_{N}^{\delta}\left(\Gamma_{\text {ref }}\right)$ of the interface, evaluate $F_{\text {star }}\left(r_{N}^{\delta}\right)$ by solving (4.4) for all incident waves $\ell=1, \ldots, m$. Save the Fourier coefficients of the interior boundary data $\boldsymbol{u}_{\ell, n_{\text {sol }}}^{i}$ and $\boldsymbol{t}_{\ell, n_{\text {sol }}}^{i}$ on $\Gamma_{N}^{\delta}$ and compute the discrete far field patterns $\boldsymbol{u}_{\ell, n_{\text {meas }}}^{\infty}$ for the $\ell$ th incident wave and the interface $\Gamma_{N}^{\delta}$ with (4.5).

If the stopping rule is not satisfied at the $N$ th regularized Gauß-Newton step, then:

3. Compute the next iterate $\boldsymbol{q}_{N+1}^{\delta}$ by minimizing the least square (4.2) (or solving the equivalent linear equation (4.3)) by the conjugate gradient method. In each CG step we need to compute $F_{\text {star }}^{\prime}\left[r_{N}^{\delta}\right] \nu$ and 
$F_{\text {star }}^{\prime}\left[r_{N}^{\delta}\right]_{\mid \boldsymbol{L}^{2}}^{*} \boldsymbol{h}$ numerous times. The Fréchet derivatives are evaluated by solving $(4.7)$ for $k=1, \ldots, m$. The right hand sides can easily be evaluated using the discrete boundary data $\left(\boldsymbol{u}_{\ell, n_{s o l}}^{i}, \boldsymbol{t}_{\ell, n_{s o l}}^{i}\right)$ (see Remark 3.2). To compute $F_{\text {star }}^{\prime}\left[\boldsymbol{q}_{N}^{\delta}\right]_{\boldsymbol{L}^{2}}^{*} \boldsymbol{h}$ with $\boldsymbol{h}={ }^{\mathrm{T}}\left(\boldsymbol{h}^{(1)}, \ldots, \boldsymbol{h}^{(m)}\right)$, we compute traces of the total fields for Herglotz incident fields with kernels $\overline{\boldsymbol{h}^{(\ell)}}$ by evaluating

$$
{ }^{\mathrm{T}}\left(-\mathcal{O}_{n_{s o l}}\left(\boldsymbol{T}_{i} \overline{\boldsymbol{u}_{\boldsymbol{q}, \overline{\boldsymbol{h}^{(\ell)}}}^{i}}\right) \circ \boldsymbol{q}, \mathcal{O}_{n_{s o l}} \overline{\boldsymbol{u}_{\boldsymbol{q}, \overline{\boldsymbol{h}^{(\ell)}}}^{i}} \circ \boldsymbol{q}\right)={ }^{\mathrm{T}} \overline{\left[\mathbf{I}_{o p}\right]_{n_{s o l}}} \mathrm{~T}_{\left(\mathscr{F}_{\mathrm{N}}, \boldsymbol{q} \quad \mathscr{F}_{\mathrm{D}}, \boldsymbol{q}\right)_{n_{\text {meas }}}} \overline{\mathbf{h}^{(\ell)}}
$$

(see proof of Proposition 3.3). The surface derivatives which appear in the formulas (3.4)-(3.6) can be computed analytically using some identities stated in [30, Section 3] or [46, Section 3] which link surfaces derivatives on $\Gamma_{\boldsymbol{q}}$ to surface derivatives on $\mathbb{S}^{2}$. The discrete analogue of the integration by part formula used in the proof of Proposition 3.3 is exact when one uses these identities.

\section{$5 \quad$ Numerical experiments}

The forward problem. We present numerical experiments for low and medium frequencies to highlight the fast convergence of the spectral algorithm [20,46] that we use to solve the elastic transmission problem. We consider various smooth convex and non convex obstacles whose the parametric representations and visualizations are given in $[19,48]$. The surfaces are characterized by their diameter denoted size_obj. The convex shapes are the sphere, denoted by sphere $($ size_obj), the ellipsoid with principal diameters $a, b$ and $c$ denoted by $\operatorname{ellipsoid}(a, b, c)$ and the rounded tetrahedron denoted by tetra $($ size_obj $)$. The non convex shape is the stellated dodecahedron denoted by $\operatorname{stell}($ size_obj).

As a first test, using the indirect approach (2.9), we compute the far field, denoted by $\boldsymbol{u}_{\mathrm{ps}}^{\infty}$, created by an off center point source located inside the elastic obstacle :

$$
\boldsymbol{u}^{i n c}(\boldsymbol{x})=-\left[\Phi^{e}(\boldsymbol{x}, \boldsymbol{s})\right] \boldsymbol{p}, \quad \boldsymbol{s} \in \Omega \text { and } \boldsymbol{p} \in \mathbb{S}^{2} .
$$

In this case the total exterior wave has to vanish so that the far-field pattern of the scattered wave $\boldsymbol{u}^{s}$ is the opposite of the far field pattern of the incident wave. The following far-field representation is obtain by applying the kernel of the far field operator $\mathscr{F}_{\mathrm{D}}$ to $\boldsymbol{p}$ (see $(2.11)$ ).

$$
\boldsymbol{u}_{\text {exact }}^{\infty}(\hat{\boldsymbol{x}})=\frac{1}{\mu_{e}} \frac{e^{-i \kappa_{s}^{e} \hat{\boldsymbol{x}} \cdot \boldsymbol{s}}}{4 \pi}(\hat{\boldsymbol{x}} \times \boldsymbol{p}) \times \hat{\boldsymbol{x}}+\frac{1}{\lambda_{e}+2 \mu_{e}} \frac{e^{-i \kappa_{p}^{e} \hat{\boldsymbol{x}} \cdot \boldsymbol{s}}}{4 \pi}(\hat{\boldsymbol{x}} \cdot \boldsymbol{p}) \hat{\boldsymbol{x}} .
$$

We choose $\boldsymbol{s}=^{\top}(0,0.05,0.0866)$ and $\boldsymbol{p}={ }^{\mathrm{T}}(1,0,0)$. In the tabulated results we indicate the uniform-norm error (by taking the maximum of errors obtained over 1300 observed directions, i.e $n_{\text {meas }}=25$ ) :

$$
\left\|\left[\boldsymbol{u}_{\mathrm{ps}}^{\infty}\right]_{n_{\text {sol }}}-\boldsymbol{u}_{\mathrm{exact}}^{\infty}\right\|_{\infty}=\max _{\hat{\boldsymbol{x}} \in \mathbb{S}^{2}}\left|\left[\boldsymbol{u}_{\mathrm{ps}}^{\infty}\right]_{n_{\text {sol }}}-\boldsymbol{u}_{\mathrm{exact}}^{\infty}\right|
$$

As a second test, using the direct approach (2.5), we compute the far-field pattern, denoted by $\boldsymbol{u}_{\text {pw }}^{\infty}$, created by the scattering of an incident plane elastic wave. In the tabulated results (excepted for the sphere) we indicate the real part and the imaginary part of the polarization component of the far-field evaluated at the incident direction : $\left[\boldsymbol{u}_{\mathrm{pw}}^{\infty}(\boldsymbol{d})\right]_{n_{\text {sol }}} \cdot \boldsymbol{p}$. In the case of the sphere we know the analytical representation of $\boldsymbol{u}_{\mathrm{pw}}^{\infty}(\boldsymbol{d})$, thus we compute the errors $\left\|\left[\boldsymbol{u}_{\mathrm{pw}}^{\infty}\right]_{n_{\text {sol }}}-\boldsymbol{u}_{\text {exact }}^{\infty}\right\|_{\infty}$ by taking the maximum errors over 1300 directions as for the point source radiation. The representation of $\boldsymbol{u}_{\mathrm{pw}}^{\infty}(\boldsymbol{d})$ is given by a series expansion of the vector spherical harmonics (see [46, Appendix] for more details). Here, we consider an incident shearing plave wave with $\boldsymbol{d}={ }^{\top}(0,0,1)$ and $\boldsymbol{p}={ }^{\top}(1,0,0)$.

In all simulations, the mechanical parameters characterizing the exterior domain are $\rho_{e}=1, \lambda_{e}=2$ and $\mu_{e}=1$ so that $\kappa_{s}^{e}=2 \kappa_{p}^{e}$. In the interior domain we set $\rho_{i}=1.3, \lambda_{i}=1.8, \mu_{i}=1.1$. The size of the scatterers 
Table 1: Solution of the forward transmission problem : numerical examples for medium and high frequency scattering.

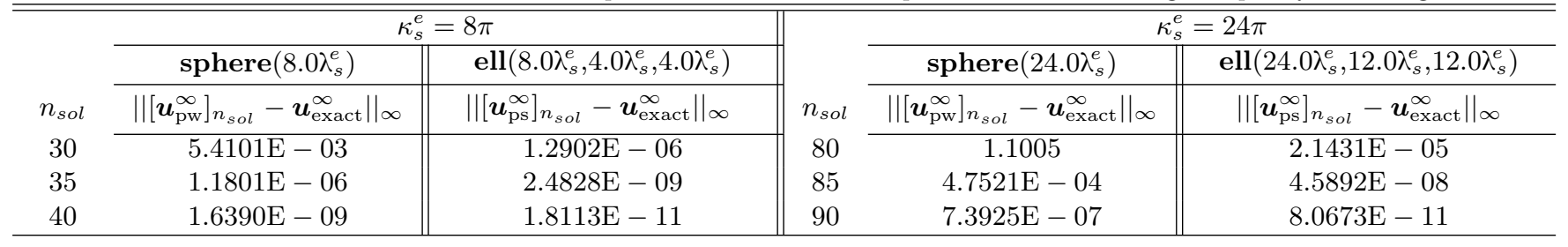

Table 2: Solution of the forward transmission problem : numerical examples for low frequency scattering

\begin{tabular}{ccccc}
\hline \hline surface & $n_{\text {sol }}$ & $\left\|\left[\boldsymbol{u}_{\mathrm{ps}}^{\infty}\right]_{n_{\text {sol }}}-\boldsymbol{u}_{\mathrm{exact}}^{\infty}\right\|_{\infty}$ & $\operatorname{Re}\left[\boldsymbol{u}_{\mathrm{pw}}^{\infty}(\boldsymbol{d})\right]_{n_{s o l}} \cdot \boldsymbol{p}$ & $\operatorname{Im}\left[\boldsymbol{u}_{\mathrm{pw}}^{\infty}(\boldsymbol{d})\right]_{n_{s o l}} \cdot \boldsymbol{p}$ \\
$\operatorname{tetra}\left(1.0290 \lambda_{s}^{e}\right)$ & 15 & $1.6623 \mathrm{E}-04$ & 0.246216901 & 0.039464026 \\
$\kappa_{s}^{e}=2$ & 25 & $1.2149 \mathrm{E}-05$ & 0.245997865 & 0.039157197 \\
& 35 & $7.3789 \mathrm{E}-07$ & 0.246014718 & 0.039116512 \\
$\operatorname{stell}\left(1.0116 \lambda_{s}^{e}\right)$ & 45 & $8.7730 \mathrm{E}-08$ & 0.246017049 & 0.039116029 \\
$\kappa_{s}^{e}=3$ & 55 & $2.0217 \mathrm{E}-04$ & 0.221257743 & 0.039813339 \\
& 70 & $1.9360 \mathrm{E}-06$ & 0.220496093 & 0.039521322 \\
& $2.9745 \mathrm{E}-07$ & 0.220449588 & 0.039547333 \\
& & & &
\end{tabular}

is indicated between brackets and is expressed in terms of the exterior S-wavelength $\lambda_{s}^{e}=2 \pi / \kappa_{s}^{e}$. As it was reported [19, 20, 46], we observe extremely fast convergence rates for smooth convex obstacles but the presence of angularity or concavity greatly increase the needed number of degree of freedom. In both cases, the spectral algorithm intially developed by Ganesh and al. $[19,20]$ is very competitive with already existing fast BEM method for low and high frequency scattering.

The Fréchet derivative. To attest the theoretical results we compare the Fréchet derivative obtained by solving the transmission problem given by Theorem 3.1 and Remark 3.2 with the Gâteaux derivative typically defined by

$$
\lim _{t \rightarrow 0} \frac{\boldsymbol{F}(\boldsymbol{q}+t \boldsymbol{\xi})-\boldsymbol{F}(\boldsymbol{q})}{t}
$$

We choose $\Gamma_{\boldsymbol{q}}=\Gamma_{\text {ref }}=\mathbb{S}^{2}$, hence $\boldsymbol{q}=\mathrm{I}$ and the object is sphere $\left(1.0 \lambda_{s}^{e}\right)$. The direction $\boldsymbol{\xi}$ and the different values of $t$ are defined in Table 3. The radial funcion $\nu$ is described by the spherical coordinates of any point $\widehat{\mathbf{x}} \in \mathbb{S}^{2}$ denoted by $(\theta, \phi) \in(0 ; \pi) \times(0 ; 2 \pi) \cup\{(0,0) ;(0, \pi)\}$. The material parameters and the incident elastic plane wave are chosen as previously with $\kappa_{s}^{e}=\pi$. We have set $n_{\text {meas }}=7, n_{\text {sol }}=10$ and $n_{\text {star }}=5$. As expected, we observe linear convergence rate.

Application to the inverse problem. The first test is concerned with the shape reconstruction problem of a convex obstacle illuminated by only one incident plane shear wave directed from top to bottom. The convex

Table 3: Numerical computation of the Fréchet derivative : comparison with the finite difference method

\begin{tabular}{c||c||cc}
\hline \hline $\boldsymbol{\xi}$ & $\boldsymbol{v}_{\boldsymbol{q}, t}^{\infty}$ & $t$ & $\left\|\boldsymbol{v}_{\boldsymbol{q}, t}^{\infty}-\boldsymbol{v}_{\boldsymbol{q}, \boldsymbol{\xi}}^{\infty}\right\|_{\infty}$ \\
\hline $\boldsymbol{\xi}=\mathcal{R} \nu$ & $\frac{\boldsymbol{u}_{\boldsymbol{q}+\boldsymbol{\xi}}^{\infty}-\boldsymbol{u}_{\boldsymbol{q}}^{\infty}}{t}$ & $\mathrm{E}-01$ & $1.7340 \mathrm{E}-01$ \\
$\nu(\widehat{\mathbf{x}})=\frac{3}{5} \cos \theta \sin \phi+\frac{2}{5} \cos 2 \theta \sin 3 \phi$ & $\mathrm{E}-03$ & $1.7344 \mathrm{E}-02$ \\
\hline
\end{tabular}


obstacle is the rounded tetrahedron whose the visualization is given in Figure 1 (a). In this case, we do not consider noisy farfield data so that $\delta=0$. We show in Figures 1 (c) and (e) some shape reconstruction for various values of the interior density $\rho_{i}$. The Lamé parameters are defined as above and we choose $\kappa_{s}^{e}=2$ so that the diameter of the scatterer is roughly $1 \lambda_{s}^{e}$. The exterior density is $\rho_{e}=1$. To compute the exact far-field data we use $n_{\text {sol }}=25$. To compute the far-field data at each iteration step we use $n_{\text {sol }}=20$. The radial functions describing the unknown parametrization belong to $\mathbb{H}_{15}^{\mathbb{R}}$. We use a mesh grid of $128\left(n_{\text {meas }}=7\right)$ degrees of freedom to evaluate the far-field pattern. The initial guess is the unit sphere. We run the algorithm until the 30-th iteration step is achieved. We choose $\alpha_{0}=10^{-2}$. We observe that the shape reconstruction of the scatterer is quite slow with a weak constrast between the exterior and interior densities and Lamé parameters. The numerical experiments show that at the 30-th iteration step with $\rho_{i}=1.3$, only the 4 vertices of the tetrahedron are recovered but the 5 edges remains concave. Increasing the constrast by considering $\rho_{i}=2$, we obtain an accurate reconstruction of the tetrahedron. Other experiments have been conducted and we found that using higher values of the frequency $\left(\kappa_{s}^{e}=3\right.$ or $\left.\kappa_{s}^{e}=4\right)$ and illuminating the scatterer by several incident plane waves (as for example 3 or 6 shear waves) greatly reduces the needed number of iterations to obtain an accurate reconstruction of the obstacle.

Our second test is concerned with the shape reconstruction of the (rounded) stellation of the dodecahedron whose the visualization is given in Figure 1 (b). This shape consists of 20 peaks located at the 20 vertices of the regular dodecahedron. In this case, we reconstruct the obstacle from noisy far-field measurements corresponding to the scattering of 6 incident plane shear waves from top, bottom, front, back, left and right sides. We consider a noise level of $5 \%$ random noise. The Lamé parameters are defined as above and we choose $\kappa_{s}^{e}=6$ so that the diameter of the scatterer is roughly $2 \lambda_{s}^{e}$. To compute the exact far-field data we use $n_{s o l}=40$. To compute the far-field data at each iteration step we use $n_{\text {sol }}=30$. The radial functions describing the unknown parametrization belong to $\mathbb{H}_{20}^{\mathbb{R}}$. The initial guess still is the unit sphere. Using the discrepancy principle, the algorithm is stopped at the first index $N$ for which

$$
\left\|F\left(\boldsymbol{q}_{N}^{\delta}\right)-\boldsymbol{u}_{\delta}^{\infty}\right\| \leq \tau \delta
$$

where we choosed $\tau=1.5$. We set again $\alpha_{0}=10^{-2}$. With this stopping rule we obtain the picture presented in Figure 1 (d) after 14 iterations and the picture presented in Figure 1 (f) after 10 iterations. We observe that noisy measurements affect the accuracy of the shape reconstruction of scatterer for weak constrast between the interior and exterior Lamé parameters and densities. One can see in Figure 1 (d) that the peaks are assembled two by two. This phenomenon is amplified by letting the algorithm running until the $\boldsymbol{L}^{2}$ error between the farfield data and the farfield pattern of the unknown scatterer is closed to $\delta$. We find that with $\rho_{i}=1.3$ the shape of the unknown scatterer stagnates and we definitively obtain the result presented in Figure 1 (d), whereas the shape reconstruction process is not finished in the case $\rho_{i}=2$ and the size of all the 20 peaks are well recovered.

These numerical experiments were realised in few hours using matlab programing language. The vector nature of elastic waves greatly increases the dimension of the integral equation systems and makes the whole algorithm significantly slower than in the case of acoustic waves. Rather recently, iterative methods for the shape reconstruction, avoiding solving the direct scattering problem at each iteration step, have drawn some interest $[35,36]$. Investigations will be conducted in this area of research. Another future research line is the combination of topological optimization tools with geometric optimization tools to find the location, the shape and the material properties of multiple elastic obstacles between interfaces of piecewise homogeneous media.

Acknowledgement. The author gratefully acknowledges Prof. Dr. Thorsten Hohage from the Institute of Numerical and Applied Mathematics at the University of Goettingen for providing the inversion toolbox he developed with Matlab programing language [28, 29]. 


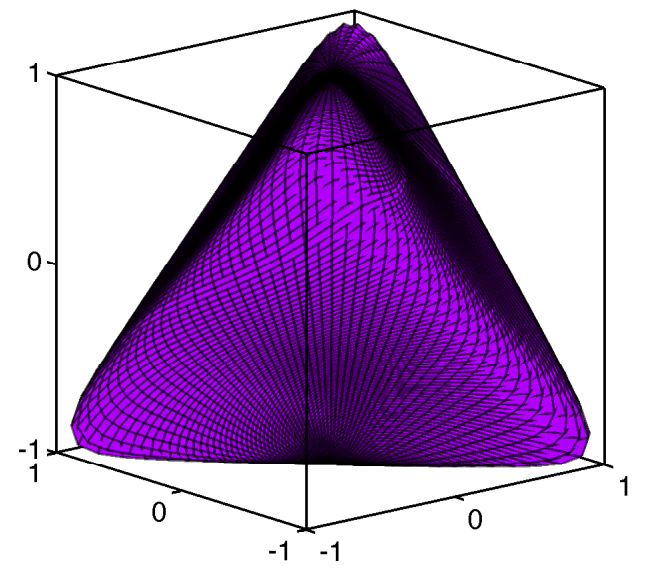

(a) true obstacle

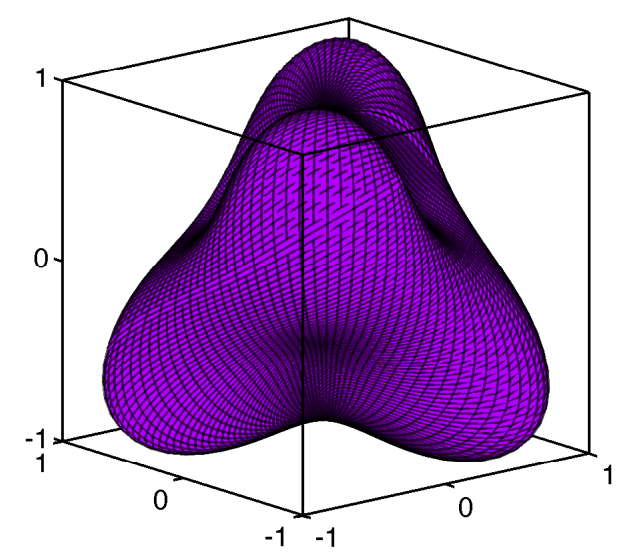

(c) 1 shear wave, $\rho_{i}=1.3,0 \%$ noise

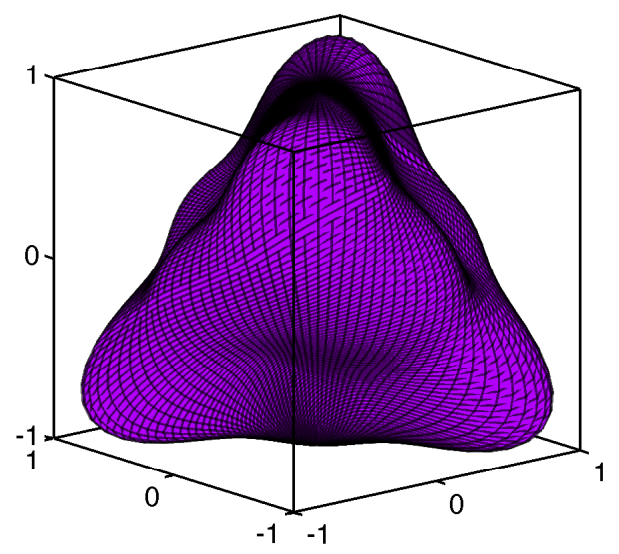

(e) 1 shear-wave, $\rho_{i}=2,0 \%$ noise

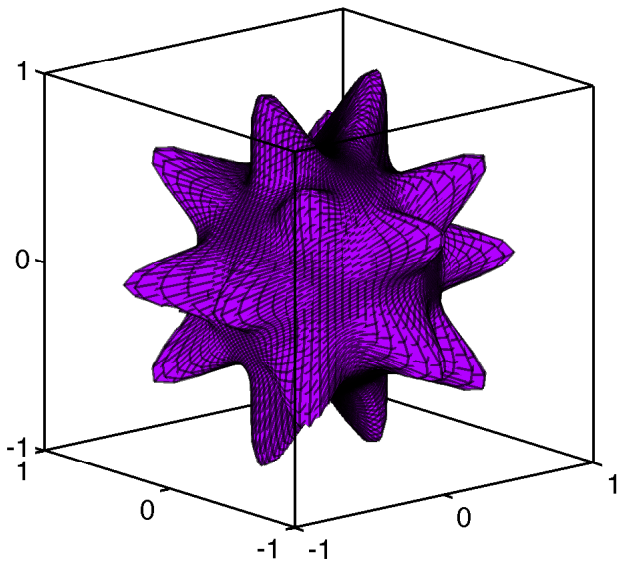

(b) true obstacle

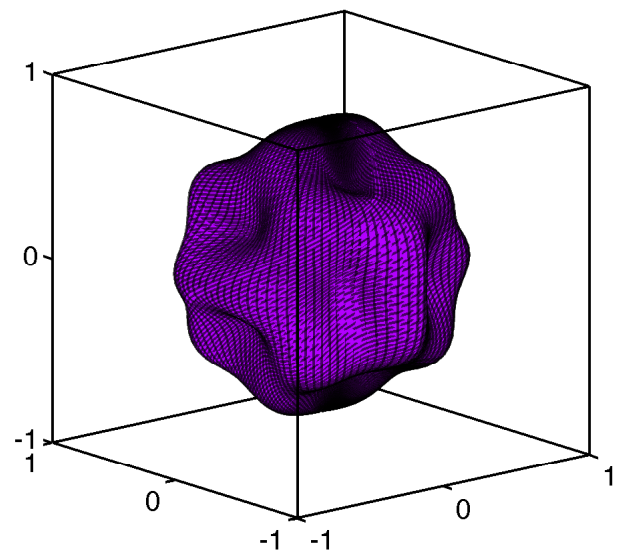

(d) 6 shear waves, $\rho_{i}=1.3,5 \%$ noise

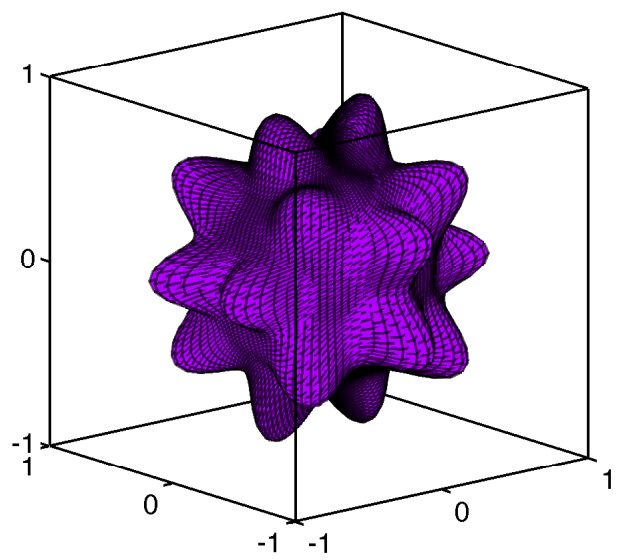

(f) 6 shear-waves, $\rho_{i}=2,5 \%$ noise

Figure 1: Iterative shape reconstruction of the tetrahedron (left side) and the stellated dodecahedron (right side). 


\section{Appendix}

This appendix is devoted to the construction of an orthonormal function basis of the Hilbert space $\boldsymbol{L}^{2}(\Gamma)$. We use the eigenfunctions of the scalar and vector Laplace-Beltrami operators following [47, Section 5.4.1]. Using this funcion basis we introduce some isomorphisms between Sobolev spaces.

Since $\Gamma$ is a compact manifold, the scalar negative Laplace-Beltrami operator $\left(-\Delta_{\Gamma}\right)$ admits a countable increasing sequence of non-negative real eigenvalues $\left(\beta_{j}\right)_{j \in \mathbb{N}}$ associated with the normalized eigenfunctions $\left(Y_{j}\right)_{j \in \mathbb{N}}$ satisfying the eigenvalue problem $-\Delta_{\Gamma} Y_{j}=\beta_{j} Y_{j}$ in $L^{2}(\Gamma)$. We have $\beta_{j} \neq 0$ for $j \geq 1$. Any scalar function $\underline{r} \in L^{2}(\Gamma)$ can be expanded on the basis $Y_{i}$ in the form

$$
\underline{r}(\boldsymbol{x})=\sum_{j=0}^{\infty} \underline{r_{j}} Y_{j}(\boldsymbol{x}), \text { with } \sum_{j=0}^{\infty}\left|\underline{r}_{j}\right|^{2}<+\infty .
$$

For any $s \in \mathbb{R}$, the space $H^{s}(\Gamma)$ of distributions can be redefined as

$$
H^{s}(\Gamma)=\left\{r ; \sum_{j=0}^{\infty}\left(1+\beta_{j}\right)^{s}\left|r_{j}\right|^{2}<\infty\right\}
$$

undowed with the norm $\|r\|_{H^{s}}^{2}=\sum_{j=0}^{+\infty}\left(1+\beta_{j}\right)^{s}\left|r_{j}\right|^{2}$. We naturally deduce the following bicontinuous isomorphism between the spaces $L^{2}(\Gamma)$ and $H^{s}(\Gamma)$ :

$$
\begin{aligned}
j_{L^{2} \rightarrow H^{s}}: \quad L^{2}(\Gamma) & \rightarrow H^{s}(\Gamma) \\
\underline{r}:=\left(\underline{r_{j}}\right)_{j \geq 0} & \mapsto r:=\left(r_{j=}\left(1+\beta_{j}\right)^{-\frac{s}{2}} \underline{r_{j}}\right)_{j \geq 0} .
\end{aligned}
$$

Since $\Delta_{\Gamma}=\operatorname{div}_{\Gamma} \nabla_{\Gamma}=-\operatorname{curl}_{\Gamma} \operatorname{curl}_{\Gamma}$, the vectors $\nabla_{\Gamma} Y_{j}$ and $\operatorname{curl}_{\Gamma} Y_{j}=\nabla_{\Gamma} Y_{j} \times \boldsymbol{n}$ are eigenvectors of the tangential vector negative Laplace-Beltrami operator $-\boldsymbol{\Delta}_{\Gamma}=\operatorname{curl}_{\Gamma} \operatorname{curl}_{\Gamma}-\nabla_{\Gamma} \operatorname{div}_{\Gamma}$ with the eigenvalue $\beta_{j}$. Let $\mathcal{N}$ be the nullspace of the vector Laplace-Beltrami operator. If the surface $\Gamma$ is simply connected then $\mathcal{N}=\{\overrightarrow{\boldsymbol{0}}\}$ otherwise, let $N_{0} \in \mathbb{N}^{*}$ be the dimension and $\left(\mathcal{Y}_{j}^{(0)}\right)_{0 \leq j<N_{0}}$ be an orthonormal function basis of $\mathcal{N}$. For $j \geq 0$ we set $\mathcal{Y}_{j}^{(3)}=\boldsymbol{n} Y_{j}$ and for $j \geq 1$ we set $\mathcal{Y}_{j}^{(1)}=\beta_{j}^{-\frac{1}{2}} \nabla_{\Gamma} Y_{j}$ and $\mathcal{Y}_{j}^{(2)}=\beta_{j}^{-\frac{1}{2}} \operatorname{curl}_{\Gamma} Y_{j}$. The concatenation of the sequences $\left(\boldsymbol{\mathcal { Y }}_{j}^{(0)}\right)_{0 \leq j<N_{0}},\left(\boldsymbol{\mathcal { Y }}_{j}^{(1)}\right)_{j \in \mathbb{N}^{*}},\left(\boldsymbol{\mathcal { Y }}_{j}^{(2)}\right)_{j \in \mathbb{N}^{*}}$ and $\left(\boldsymbol{\mathcal { Y }}_{j}^{(3)}\right)_{j \in \mathbb{N}}$ forms an orthonormal basis of $\boldsymbol{L}^{2}(\Gamma)$.

Any vector dentity $\varphi \in \boldsymbol{L}^{2}(\Gamma)$ can be expanded in the above eigenvector basis as follows

$$
\boldsymbol{\varphi}=\sum_{j=0}^{N_{0}-1} \underline{\varphi_{j}^{(0)}} \mathcal{Y}_{j}^{(0)}+\sum_{j=1}^{+\infty}\left[\underline{\varphi_{j}^{(1)}} \mathcal{Y}_{j}^{(1)}+\underline{\varphi_{j}^{(2)}} \mathcal{Y}_{j}^{(2)}\right]+\sum_{j=0}^{+\infty} \underline{\varphi_{j}^{(3)}} \mathcal{Y}_{j}^{(3)}
$$

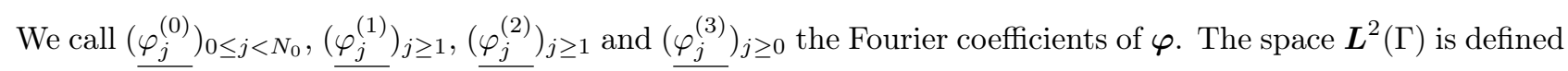
by

$$
\boldsymbol{L}^{2}(\Gamma):=\left\{\boldsymbol{\varphi} ;\left|\underline{\varphi_{0}^{(3)}}\right|^{2}+\sum_{j=0}^{N_{0}-1}\left|\underline{\varphi_{j}^{(0)}}\right|^{2}+\sum_{j=1}^{+\infty}\left[\left|\underline{\mid \varphi_{j}^{(1)}}\right|^{2}+\left|\underline{\varphi_{j}^{(2)}}\right|^{2}+\left|\underline{\varphi_{j}^{(3)}}\right|^{2}\right]<+\infty\right\},
$$

undowed with the norm $\|\varphi\|_{L^{2}}^{2}=\left|\underline{\varphi_{0}^{(3)}}\right|^{2}+\sum_{j=0}^{N_{0}-1}\left|\underline{\varphi_{j}^{(0)}}\right|^{2}+\sum_{j=1}^{+\infty}\left[\left|\underline{\varphi_{j}^{(1)}}\right|^{2}+\left|\underline{\varphi_{j}^{(2)}}\right|^{2}+\left|\underline{\varphi_{j}^{(3)}}\right|^{2}\right]$. For any $s \in \mathbb{R}$ we define the vector space $\boldsymbol{H}^{s}(\Gamma)$ by (extending [47, (5.4.17)] to non tangential fields)

$$
\boldsymbol{H}^{s}(\Gamma):=\left\{\boldsymbol{\varphi} ;\left|\varphi_{0}^{(3)}\right|^{2}+\sum_{j=0}^{N_{0}-1}\left|\varphi_{j}^{(0)}\right|^{2}+\sum_{j=1}^{+\infty}\left(\beta_{j}\right)^{s}\left[\left|\varphi_{j}^{(1)}\right|^{2}+\left|\varphi_{j}^{(2)}\right|^{2}+\left|\varphi_{j}^{(3)}\right|^{2}\right]<+\infty\right\},
$$


undowed with the norm $\|\varphi\|_{\boldsymbol{H}^{s}}^{2}=\left|\varphi_{0}^{(3)}\right|^{2}+\sum_{j=0}^{N_{0}-1}\left|\varphi_{j}^{(0)}\right|^{2}+\sum_{j=1}^{+\infty}\left(\beta_{j}\right)^{s}\left[\left|\varphi_{j}^{(1)}\right|^{2}+\left|\varphi_{j}^{(2)}\right|^{2}+\left|\varphi_{j}^{(3)}\right|^{2}\right]$. We naturally deduce the following bicontinuous isomorphism between the spaces $\boldsymbol{L}^{2}(\Gamma)$ and $\boldsymbol{H}^{s}(\Gamma)$ :

$$
\begin{aligned}
& j_{\boldsymbol{L}^{2} \rightarrow \boldsymbol{H}^{s}}: \quad \boldsymbol{L}^{2}(\Gamma) \quad \rightarrow \quad \boldsymbol{H}^{s}(\Gamma)
\end{aligned}
$$

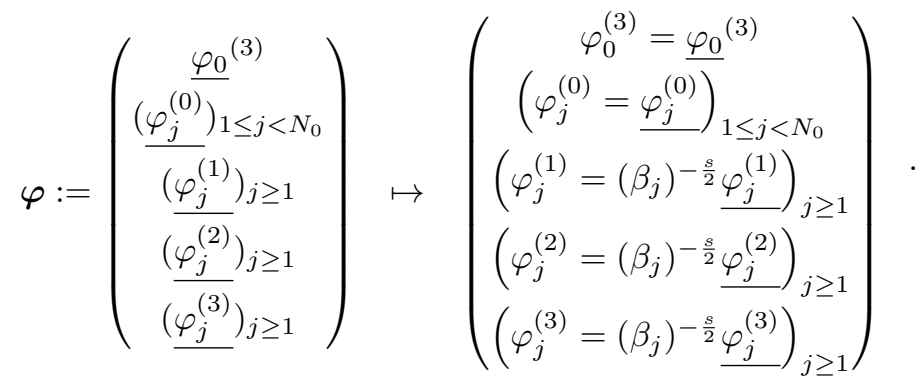

In the special case $\Gamma=\mathbb{S}^{2}$, the expression of the eigenvalues and the eigenfunctions of the scalar and vector Laplace Beltrami operator are well-known. The exact expressions are given for instance in [30, Appendix B].

\section{References}

[1] R. A. Adams, Sobolev spaces, Academic Press [A subsidiary of Harcourt Brace Jovanovich, Publishers], New YorkLondon, 1975. Pure and Applied Mathematics, Vol. 65.

[2] M. S. Agranovich, B. A. Amosov, And M. Levitin, Spectral problems for the Lamé system with spectral parameter in boundary conditions on smooth or nonsmooth boundary, Russ. J. Math. Phys., 6 (1999), pp. $247-281$.

[3] C. J. S. Alves And R. Kress, On the far-field operator in elastic obstacle scattering, IMA J. Appl. Math., 67 (2002), pp. 1-21.

[4] K. Baganas, B. B. Guzina, A. Charalambopoulos, and G. D. Manolis, A linear sampling method for the inverse transmission problem in near-field elastodynamics, Inverse Problems, 22 (2006), pp. 1835-1853.

[5] A. B. Bakushinskil, On a convergence problem of the iterative-regularized Gauss-Newton method, Comput. Math. Math. Phys, 32 (1992), pp. 1503-1509.

[6] L. Bourgeois, F. Le Louër, And E. Lunéville, On the use of Lamb modes in the linear sampling method for elastic waveguides, Inverse Problems, 27 (2011), pp. 055001, 27.

[7] L. Bourgeois And E. LunÉville, On the use of the linear sampling method to identify cracks in elastic waveguides, Inverse Problems, 29 (2013), pp. 025017, 19.

[8] A. Carpio, B. T. Johansson, And M.-L. Rapún, Determining planar multiple sound-soft obstacles from scattered acoustic fields, J. Math. Imaging Vision, 36 (2010), pp. 185-199.

[9] A. Charalambopoulos, On the Fréchet differentiability of boundary integral operators in the inverse elastic scattering problem, Inverse Problems, 11 (1995), pp. 1137-1161.

[10] A. Charalambopoulos, D. Gintides, and K. Kiriaki, The linear sampling method for the transmission problem in three-dimensional linear elasticity, Inverse Problems, 18 (2002), pp. 547-558.

[11] A. Charalambopoulos, A. Kirsch, K. A. Anagnostopoulos, D. Gintides, and K. Kiriaki, The factorization method in inverse elastic scattering from penetrable bodies, Inverse Problems, 23 (2007), pp. 27-51.

[12] D. Colton And A. Kinsch, A simple method for solving inverse scattering problems in the resonance region, Inverse Problems, 12 (1996), pp. 383-393.

[13] D. Colton, M. Piana, And R. Potthast, A simple method using Morozov's discrepancy principle for solving inverse scattering problems, Inverse Problems, 13 (1997), pp. 1477-1493.

[14] D. L. Colton And R. Kress, Inverse acoustic and electromagnetic scattering theory, vol. 93 of Applied Mathematical Sciences, Springer-Verlag, Berlin, second ed., 1998. 
[15] M. Costabel And F. Le Louër, Shape derivatives of boundary integral operators in electromagnetic scattering. Part I: Shape differentiability of pseudo-homogeneous boundary integral operators, Integral Equations and Operator Theory, 72 (2012), pp. 509-535.

[16] - Shape derivatives of boundary integral operators in electromagnetic scattering. Part II: Application to scattering by a homogeneous dielectric obstacle, Integr.Equ.Oper.Theory, 73 (2012), pp. 17-48.

[17] M. Costabel and E. P. Stephan, Integral equations for transmission problems in linear elasticity, J. Integral Equations Appl., 2 (1990), pp. 211-223.

[18] M. Darbas And F. Le LouËR, Well-conditioned boundary integral formulations for the iterative solution of elastic scattering problems, Math. Meth. Appl. Sci., 38 (2015), pp. 1705-1733.

[19] M. Ganesh and I. G. Graham, A high-order algorithm for obstacle scattering in three dimensions, J. Comput. Phys., 198 (2004), pp. 211-242.

[20] M. Ganesh And S. C. Hawkins, A hybrid high-order algorithm for radar cross section computations, SIAM J. Sci. Comput., 29 (2007), pp. 1217-1243.

[21] H. HADdAR AND R. KRess, On the Fréchet derivative for obstacle scattering with an impedance boundary condition, SIAM J. Appl. Math., 65 (2004), pp. 194-208 (electronic).

[22] P. Hähner And G. C. Hsiao, Uniqueness theorems in inverse obstacle scattering of elastic waves, Inverse Problems, 9 (1993), pp. 525-534.

[23] H. Harbrecht and T. Hohage, Fast methods for three-dimensional inverse obstacle scattering problems, J. Integral Equations Appl., 19 (2007), pp. 237-260.

[24] F. Hettlich, Fréchet derivatives in inverse obstacle scattering, Inverse Problems, 11 (1995), pp. 371-382.

[25] _ Erratum: "Frechet derivatives in inverse obstacle scattering" [Inverse Problems 11 (1995), no. 2, 371-382; MR1324650 (95k:35217)], Inverse Problems, 14 (1998), pp. 209-210.

[26] - The domain derivative of time-harmonic electromagnetic waves at interfaces, Math. Methods Appl. Sci., 35 (2012), pp. 1681-1689.

[27] F. Hettlich and W. Rundell, A second degree method for nonlinear inverse problems, SIAM J. Numer. Anal., 37 (2000), pp. 587-620.

[28] T. Hohage, Logarithmic convergence rates of the iteratively regularized Gauss-Newton method for an inverse potential and an inverse scattering problem, Inverse Problems, 13 (1997), pp. 1279-1299.

[29] — Iterative Methods in Inverse Obstacle Scattering: Regularization Theory of Linear and Nonlinear Exponentially Ill-Posed Problems, PhD thesis, University of Linz, 1999.

[30] T. Hohage And F. Le Louër, A spectrally accurate method for the dielectric obstacle scattering problem and applications to the inverse problem, preprint, (2013). http://num.math.uni-goettingen.de/preprints/files/2013-20.pdf.

[31] T. Hohage and C. Schormann, A Newton-type method for a transmission problem in inverse scattering, Inverse Problems, 14 (1998), pp. 1207-1227.

[32] G. C. Hsiao and W. L. Wendland, Boundary integral equations, vol. 164 of Applied Mathematical Sciences, Springer-Verlag, Berlin, 2008.

[33] G. Hu, A. Kirsch, And M. Sini, Some inverse problems arising from elastic scattering by rigid obstacles, Inverse Problems, 29 (2013), pp. 015009, 21.

[34] G. Hu, Y. Lu, AND B. Zhang, The factorization method for inverse elastic scattering from periodic structures, Inverse Problems, 29 (2013), pp. 115005, 25.

[35] O. Ivanyshyn and T. Johansson, Nonlinear integral equation methods for the reconstruction of an acoustically sound-soft obstacle, J. Integral Equations Appl., 19 (2007), pp. 289-308.

[36] O. Ivanyshyn and R. Kress, Identification of sound-soft 3D obstacles from phaseless data, Inverse Probl. Imaging, 4 (2010), pp. 131-149.

[37] B. Kaltenbacher and B. Hofmann, Convergence rates for the iteratively regularized Gauss-Newton method in Banach spaces, Inverse Problems, 26 (2010), pp. 035007, 21. 
[38] A. KIRsch, The domain derivative and two applications in inverse scattering theory, Inverse Problems, 9 (1993), pp. $81-96$.

[39] A. KIRsch, Characterization of the shape of a scattering obstacle using the spectral data of the far field operator, Inverse Problems, 14 (1998), pp. 1489-1512.

[40] R. E. Kleinman and P. A. Martin, On single integral equations for the transmission problem of acoustics, SIAM J. Appl. Math., 48 (1988), pp. 307-325.

[41] R. KRess, Electromagnetic waves scattering : Scattering by obstacles, Scattering, (2001), pp. 191-210. Pike, E. R. and Sabatier, P. C., eds., Academic Press, London.

[42] R. Kress and L. PÄIVÄrinta, On the far field in obstacle scattering, SIAM J. Appl. Math., 59 (1999), pp. 14131426 (electronic).

[43] R. Kress and G. F. Roach, Transmission problems for the helmholtz equation, J. Math. Phys., 19 (1978), pp. 14331437.

[44] V. D. Kupradze, T. G. Gegelia, M. O. Basheleřshvili, and T. V. Burchuladze, Three-dimensional problems of the mathematical theory of elasticity and thermoelasticity, vol. 25 of North-Holland Series in Applied Mathematics and Mechanics, North-Holland Publishing Co., Amsterdam, russian ed., 1979. Edited by V. D. Kupradze.

[45] F. LE LouËr, On the Fréchet derivative in elastic obstacle scattering, SIAM J. Appl. Math., 72 (2012), pp. 14931507.

[46] _ A high order spectral algorithm for elastic obstacle scattering in three dimensions, J. Comput. Phys., 279 (2014), pp. 1-17.

[47] J.-C. NÉDÉLEC, Acoustic and electromagnetic equations, vol. 144 of Applied Mathematical Sciences, Springer-Verlag, New York, 2001. Integral representations for harmonic problems.

[48] S. ONAKA, Simple equations giving shapes of various convex polyhedra: The regular polydedra and polyhedra composed of crystallographically low-index planes, Philosophical Magazine Letters, 86 (2006), pp. 175-183.

[49] R. Potтhast, Fréchet differentiability of boundary integral operators in inverse acoustic scattering, Inverse Problems, 10 (1994), pp. 431-447.

[50] - Domain derivatives in electromagnetic scattering, Math. Methods Appl. Sci., 19 (1996), pp. 1157-1175. 